\title{
Representation theory for the Križ model
}

\author{
SAMIA ASHRAF \\ HANIYA AZAM \\ BARBU BERCEANU
}

The natural action of the symmetric group on the configuration spaces $F(X, n)$ induces an action on the Križ model $E(X, n)$. The representation theory for this complex is studied and a big acyclic subcomplex which is $\mathcal{S}_{n}$-invariant is described.

55R80, 20C30; 55P62, 13A50

\section{Introduction}

The ordered configuration space of $n$ points $F(X, n)$ of a topological space $X$ is defined as

$$
F(X, n)=\left\{\left(x_{1}, x_{2}, \ldots, x_{n}\right) \in X^{n} \mid x_{i} \neq x_{j} \text { for } i \neq j\right\} .
$$

For $X$ a smooth complex projective variety, I Križ [16] constructed a rational model $E(X, n)$ for $F(X, n)$, a simplified version of the Fulton-MacPherson model [15].

Let us recall the construction of Križ. We denote by $p_{i}^{*}: H^{*}(X) \rightarrow H^{*}\left(X^{n}\right)$ and $p_{i j}^{*}: H^{*}\left(X^{2}\right) \rightarrow H^{*}\left(X^{n}\right)$ (for $i \neq j$ ) the pullbacks of the obvious projections and by $m$ the complex dimension of $X$ (for cohomology groups we use rational or complex coefficients). The model $E(X, n)$ is defined as follows: as an algebra $E(X, n)$ is isomorphic to the exterior algebra with generators $G_{i j}, 1 \leq i, j \leq n$ (of degree $2 m-1$ ) and coefficients in $H^{*}(X)^{\otimes n}$ modulo the relations

$$
\begin{aligned}
G_{j i} & =G_{i j}, & & \\
p_{j}^{*}(x) G_{i j} & =p_{i}^{*}(x) G_{i j}, & & i<j, x \in H^{*}(X), \\
G_{i k} G_{j k} & =G_{i j} G_{j k}-G_{i j} G_{i k}, & & i<j<k .
\end{aligned}
$$

The differential $d$ is given by $\left.d\right|_{H^{*}(X)} \otimes n=0$ and $d\left(G_{i j}\right)=p_{i j}^{*}(\Delta)$, where $\Delta$ denotes the class of the diagonal $w \otimes 1+\cdots+1 \otimes w \in H^{*}(X) \otimes H^{*}(X)$ and $w \in H^{2 m}(X)$ is the fundamental class.

This model is a differential bigraded algebra $E(X, n)=\bigoplus_{k, q} E_{q}^{k}(X, n)$ : the lower degree $q$ (called the exterior degree) is given by the number of exterior generators $G_{i j}$, 
and the upper degree $k$ is given by the total degree; the multiplication is homogeneous and the differential has bidegree $\left(\begin{array}{l}+1 \\ -1\end{array}\right)$ :

$$
E_{q}^{k} \otimes E_{q^{\prime}}^{k^{\prime}} \rightarrow E_{q+q^{\prime}}^{k+k^{\prime}}, \quad d: E_{q}^{k} \rightarrow E_{q-1}^{k+1}
$$

In the next definition $G_{I_{*} J_{*}}$ is a product of exterior generators

$$
G_{I_{*} J_{*}}=G_{i_{1} j_{1}} G_{i_{2} j_{2}} \cdots G_{i_{q} j_{q}} .
$$

Definition 1.1 (Fulton and MacPherson [15], Križ [16]) The symmetric group $\mathcal{S}_{n}$ acts on $E(X, n)$ by permuting the factors in $H^{*}\left(X^{n}\right)=H^{* \otimes n}$ and changing the indices of the exterior generators: for an arbitrary permutation $\sigma \in \mathcal{S}_{n}$,

$$
\sigma\left(p_{1}^{*}\left(x_{h_{1}}\right) \cdots p_{n}^{*}\left(x_{h_{n}}\right) G_{I_{*} J_{*}}\right)=p_{\sigma(1)}^{*}\left(x_{h_{1}}\right) \cdots p_{\sigma(n)}^{*}\left(x_{h_{n}}\right) G_{\sigma\left(I_{*}\right) \sigma\left(J_{*}\right)} \text {. }
$$

The action of $\mathcal{S}_{n}$ is well-defined because the set of relations is invariant under this action.

In the next diagram the nonzero bigraded components $E_{q}^{k}(X, n)$ lie in the trapezoid with vertices $(0,0),(2 m n, 0),((n-1)(2 m-1), n-1)$ and $(n(2 m-1)+1, n-1)$. The arrows show the direction of the differentials $(+1,-1)$ :

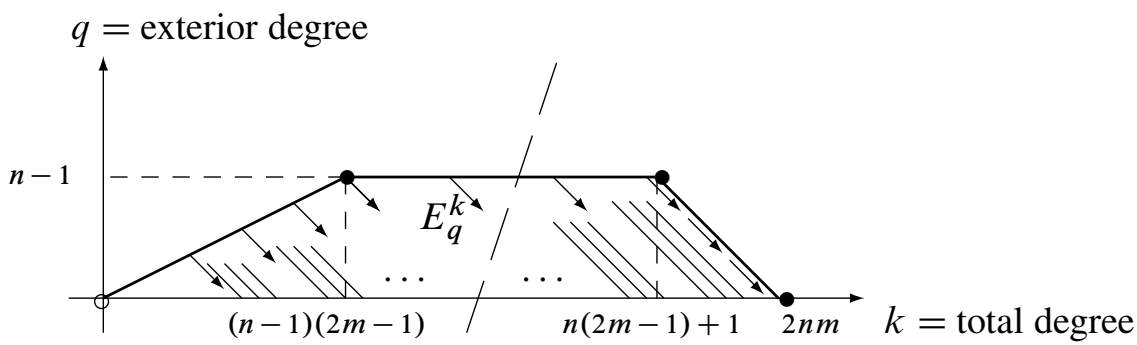

For each $q$ in the interval $[0, n-1]$ in the horizontal graded module $E_{q}^{*}=\bigoplus_{k} E_{q}^{k}$ the components equally distanced from the dotted median line are isomorphic as $\mathcal{S}_{n}$-modules; see Proposition 2.2 in Section 2. In the same section we introduce the combinatorial "types" of monomials of $E_{*}^{*}(X, n)$ : these are parameterized by forests in which every tree contains a cohomology class of $X$. The type decomposition of the bigraded components gives a direct sum of $\mathcal{S}_{n}$ submodules, each of these being generated by a unique element; see Theorem 2.13. In the next section we describe the $\mathcal{S}_{n}$ structure of types: explicit decomposition into irreducible representations in many particular cases and, using the results of Lehrer and Solomon [19], we compute the character for the general type. See Propositions 3.1, 3.2, 3.3, 3.4 and Theorem 3.11. 
In Section 4 we present some properties of the differential which are consequences of its $\mathcal{S}_{n}$-equivariance. For all smooth projective varieties, except the projective line, we show that the differential is injective on the "left side" of the trapezoid (the component $E_{0}^{0} \cong \mathbb{Q}$ contributes to the cohomology group $\left.H^{0}(F(X, n) ; \mathbb{Q}) \cong \mathbb{Q}\right)$.

Proposition 1.1 The differentials in the Križ model of a smooth complex projective variety different from $\mathbb{C} P^{1}$ are injective for any $q$ in the interval $[1, n-1]$ :

$$
d: E_{q}^{q(2 m-1)}(X, n) \succ E_{q-1}^{q(2 m-1)+1}(X, n)
$$

The top horizontal line has no contribution to the cohomology either, thus we have the following.

Proposition 1.2 The top differentials in the Križ model are injective for any $k$ in the interval $[(n-1)(2 m-1), n(2 m-1)+1]$ :

$$
d: E_{n-1}^{k}(X, n) \succ E_{n-2}^{k+1}(X, n)
$$

In Section 5 we show that the "right side" of the trapezoid is an acyclic complex.

Proposition 1.3 All cohomology groups of the subcomplex

$$
0 \rightarrow E_{n-1}^{n(2 m-1)+1}(X, n) \rightarrow E_{n-2}^{n(2 m-1)+2}(X, n) \rightarrow \cdots \rightarrow E_{0}^{2 n m}(X, n) \rightarrow 0
$$

are zero.

Other (smaller) copies of this subcomplex are contained in the interior of the trapezoid and their sum gives a large acyclic complex which is also $\mathcal{S}_{n}$-equivariant. This subcomplex $E_{*}^{*}(w(X, n))$ and the quotient $E_{*}^{*}(X, n) / E_{*}^{*}(w(X, n))$ are described in Propositions 5.5 and 5.6; the location of the subcomplex $E_{*}^{*}(w(X, n))$ is given in the diagram by the interior lines with slope -1. In [6] a different acyclic subcomplex of $E_{*}^{*}(X, n)$ is described by the third author, Markl and Papadima: this is an ideal, but is not an $\mathcal{S}_{n}$-submodule. The subcomplex $E_{*}^{*}(w(X, n))$ is an $\mathcal{S}_{n}$-subalgebra, but not an ideal. The right side of the trapezoid, denoted by $E_{*}^{\mathrm{Top}}(X, n)$ in Section 5 , is an acyclic ideal which is also an $\mathcal{S}_{n}$-submodule, but it is quite small.

In the last section the simplest and, from the viewpoint of Proposition 1.1, the exceptional case of $\mathbb{C} P^{1}$ is analyzed; we recover and we complete the results of Cohen and Taylor [11; 10] and Feichtner and Ziegler [13], computing in this case the Poincaré polynomials in two variables:

$$
P_{F(X, n)}(t, s)=\sum_{k, q \geq 0}\left(\operatorname{dim} H_{q}^{k}\right) t^{k}{ }_{s} q
$$


Theorem 1.2 In the cohomology algebra of the configuration space $F\left(\mathbb{C} P^{1}, n\right)(n \geq 4)$ the nonzero bigraded components are

$$
H_{q}^{q} \cong H_{q+1}^{q+3} \quad \text { for } q=0,1, \ldots, n-3 .
$$

Its Poincaré polynomial is

$$
P_{F\left(\mathbb{C} P^{1}, n\right)}(t, s)=\left(1+s t^{3}\right)(1+2 s t)(1+3 s t) \cdots(1+(n-2) s t) .
$$

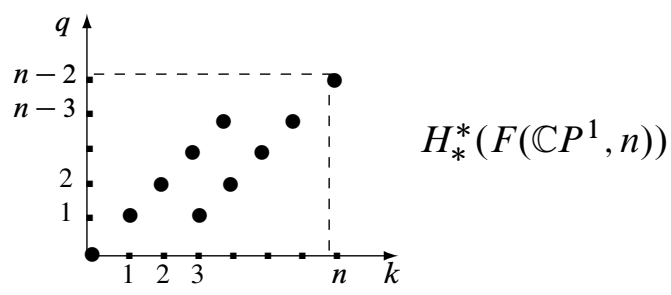

For the irreducible $\mathcal{S}_{n}$-modules we will use the standard notation (see Fulton and Harris [14]): $V(\lambda)$ corresponds to the partition of $n, \lambda \vdash n, \lambda=\left(\lambda_{1} \geq \lambda_{2} \geq \cdots \geq\right.$ $\lambda_{t} \geq 1$ ), and also the stable notation (see Church and Farb [9] or the authors [2]): $V(\mu)_{n}=V\left(n-\sum \mu_{i}, \mu_{1}, \mu_{2}, \ldots, \mu_{s}\right)$ for $\mu=\left(\mu_{1} \geq \mu_{2} \geq \cdots \geq \mu_{s} \geq 1\right)$ satisfying the relation $n-\sum_{i=1}^{s} \mu_{i} \geq \mu_{1}$.

Other extensions and applications of the results of this paper could be found by the first and third authors in [3;4] and the second and third authors [5].

Recently Lambrechts and Stanley [17] constructed a (quasi)model for the configuration space of a topological space with Poincare duality cohomology; if such a space is formal, the model of Lambrechts and Stanley is reduced to the Križ model and this is the case of Kähler manifolds; see Deligne, Griffiths, Morgan and Sullivan [12]. The results of this paper could be applied to (simply connected) formal closed manifolds (with few changes for the odd-dimensional manifolds).

Acknowledgements This research is partially supported by Higher Education Commission, Pakistan.

\section{Cohomology classes in the forest}

We will now fix a (monomial) ordered basis for the cohomology algebra $H^{*}(X ; \mathbb{Q})$ : $\mathcal{B}=\left\{x_{1}=1 \prec x_{2} \prec \cdots \prec x_{B}=w\right\}$, where $w \in H^{2 m}(X ; \mathbb{Q})$ is the fundamental class of $X$ and $B=\sum \beta_{i}$ is the sum of Betti numbers; we choose the order $\prec$ such that 
the sequence $\left\{\operatorname{deg} x_{i}\right\}_{i=1, B}$ is increasing (not necessarily strictly increasing). Using simple computations with Diamond lemma (see Bergman [7]) one can find a monomial basis for the Križ model: we denote by $G_{I_{*} J_{*}}=G_{i_{1} j_{1}} G_{i_{2} j_{2}} \cdots G_{i_{q} j_{q}}$ the exterior monomial corresponding to the sequences $I_{*}=\left(i_{1}, \ldots, i_{q}\right), J_{*}=\left(j_{1}, \ldots, j_{q}\right)$, where $i_{a}<j_{a}(a=1,2, \ldots, q)$ and $j_{1}<j_{2}<\cdots<j_{q}$, and by $x_{H_{*}}=x_{h_{1}} \otimes x_{h_{2}} \otimes \cdots \otimes x_{h_{n}}$ $\left(x_{h_{a}} \in \mathcal{B}\right)$ a scalar from $H^{* \otimes n}$. Then

$$
\left\{x_{H_{*}} G_{I_{*} J_{*}} \mid x_{h_{a}}=1 \text { if } a \in J_{*}, \operatorname{deg} x_{H_{*}}=k-q(2 m-1)\right\}
$$

is a basis of $E_{q}^{k}(X, n)$ and we call it the canonical (Bezrukavnikov) basis (see Bezrukavnikov [8]).

The next result is obvious.

Proposition 2.1 The bigraded components $E_{q}^{k}(X, n)$ are invariant under the action of the symmetric group and the differential $d$ is $\mathcal{S}_{n}$-equivariant:

$$
d\left(\sigma\left(x_{H_{*}} G_{I_{*} J_{*}}\right)\right)=\sigma\left(d\left(x_{H_{*}} G_{I_{*} J_{*}}\right)\right)
$$

This proposition and the Schur lemma give a splitting of the Križ complex into subcomplexes corresponding to the decomposition of $E_{*}^{*}$ into isotypic components $E_{*}^{*}(V(\lambda))$, for $\lambda$ an arbitrary partition of $n$ :

$$
\left(E_{*}^{*}(X, n), d\right)=\bigoplus_{\lambda \vdash n}\left(E_{*}^{*}(V(\lambda)), d_{\lambda}\right)
$$

The cohomology algebra $H^{*}(X ; \mathbb{Q})$ satisfies Poincaré duality; denote by $\mathcal{B}^{*}$ the Poincaré dual basis

$$
\mathcal{B}^{*}=\left\{y^{1}=w, y^{2}, \ldots, y^{B}=1 \mid \text { if } \operatorname{deg} x_{i}+\operatorname{deg} y^{j}=2 m, \text { then } x_{i} y^{j}=\delta_{i j} w\right\} .
$$

Proposition 2.2 For any $q=0,1, \ldots, n-1$ and any $k$ in the interval of integers $[(2 m-1) q, 2 m n-q]$, there is an isomorphism of $\mathcal{S}_{n}$-modules

$$
E_{q}^{k}(X, n) \cong E_{q}^{2 m n+2 q(m-1)-k}(X, n) .
$$

Proof Define the map $\Phi: E_{q}^{k} \rightarrow E_{q}^{2 m n+2 q(m-1)-k}$ on the basis by

$$
\Phi\left(x_{H_{*}} G_{I_{*} J_{*}}\right)=x_{H_{*}}^{\prime} G_{I_{*} J_{*}},
$$

where the factors of $x_{H_{*}}^{\prime}=x_{h_{1}}^{\prime} \otimes x_{h_{2}}^{\prime} \otimes \cdots \otimes x_{h_{n}}^{\prime}$ are given by

$$
x_{h_{a}}^{\prime}= \begin{cases}1\left(=x_{h_{a}}\right) & \text { if } h_{a} \in J_{*}, \\ y^{h_{a}} & \text { if } h_{a} \text { is not in } J_{*} .\end{cases}
$$


It is easy to see that $\Phi$ is $\mathcal{S}_{n}$-equivariant and the sum of the total degree of $x_{H_{*}} G_{I_{*} J_{*}}$ and the total degree of $\Phi\left(x_{H_{*}} G_{I_{*} J_{*}}\right)$ is $2 m n+2 q(m-1)$.

In [19] Lehrer and Solomon studied the representation theory of the Arnold algebra $\mathcal{A}^{*}(n)$, the cohomology algebra of $F(\mathbb{C}, n)$ (see Arnold [1]).

Definition 2.1 The Arnold algebra $\mathcal{A}^{*}(n)$ is defined by

$$
\mathcal{A}^{*}(n)=\bigwedge\left(G_{i j}, 1 \leq i<j \leq n\right) /\left(G_{i j} G_{i k}-G_{i j} G_{j k}+G_{i k} G_{j k}\right),
$$

where the generators $G_{i j}$ have degree 1 .

In Section 5 we will define a differential on $\mathcal{A}^{*}(n)$.

A basis of this algebra is given by monomials $G_{I_{*} J_{*}}=G_{i_{1} j_{1}} G_{i_{2} j_{2}} \cdots G_{i_{q} j_{q}}$, where $2 \leq j_{1}<j_{2}<\cdots<j_{q} \leq n$ and $1 \leq i_{a}<j_{a}$ for any $a=1, \ldots, q$. The symmetric group $\mathcal{S}_{n}$ acts naturally on $\mathcal{A}^{*}(n): \sigma . G_{I_{*} J_{*}}=G_{\sigma\left(I_{*}\right) \sigma\left(J_{*}\right)}$. To simplify the proofs, Lehrer and Solomon associated graphs to the monomials in $\mathcal{A}^{*}(n)$ : to any monomial $G_{I_{*} J_{*}}$ from the Arnold algebra $\mathcal{A}^{*}(n)$, they associated the graph $\gamma$ with vertices $\{1,2, \ldots, n\}$ and edges $\{i, j\}$ corresponding to the factors $G_{i j}$ of the given monomial.

Conversely, to any simple graph $\gamma$ (ie no double edges, no loops) on the set $\{1,2, \ldots, n\}$ one can associate an element of $\mathcal{A}^{*}(n)$ : to the graph without edges we associate $1 \in \mathcal{A}^{*}(n)$; otherwise to any edge $\{i, j\}$ we consider the factor $G_{i j}$ and take their product in the lexicographic order.

Remark 2.2 [19] If the graph $\gamma$ contains a cycle, the associated element in $\mathcal{A}^{*}(n)$ is zero.

Proof Start an induction on the length of the cycle with length 3:

$$
G_{i j} G_{i k} G_{j k}=G_{i j}\left(G_{i j} G_{j k}-G_{i j} G_{i k}\right)=0
$$

To a cycle of length $l+1$ corresponds an element containing as a factor the product

$$
G_{i_{1} i_{2}} G_{i_{2} i_{3}} \cdots G_{i_{l} i_{l+1}} G_{i_{l+1} i_{1}}=G_{i_{1} i_{2}} \cdots G_{i_{l-1} i_{l}}\left(G_{i_{1} i_{l}} G_{i_{1} i_{l+1}}-G_{i_{1} i_{l}} G_{i_{l} i_{l+1}}\right),
$$

both terms having associated graphs with cycles of length $l$.

Now we extend Lehrer and Solomon's construction to the Križ model: we add to their construction "marks" which are elements of a (fixed) monomial basis of the cohomology algebra $H^{*}(X ; \mathbb{Q})$. 
Definition 2.3 We associate to the monomial $\mu=p_{1}^{*}\left(x_{h_{1}}\right) \cdots p_{n}^{*}\left(x_{h_{n}}\right) G_{I_{*} J_{*}}$ from the canonical basis of $E_{*}^{*}(X, n)$ :

(a) The Lehrer-Solomon graph $\gamma$ associated to $G_{I_{*} J_{*}}$ : vertices $1, \ldots, n$ and for each factor $G_{i j}$ we take the edge $\{i, j\}$.

(b) To each connected component of this $\gamma$ we associate the "mark" $x_{h_{i}}$, the cohomology class lying on the position $i$, where $i$ is the smallest index in the given connected component.

Example 2.4 (a) Consider the monomial $\mu_{0}=G_{I_{*} J_{*}}=G_{12} G_{23} G_{45} G_{46} G_{47} G_{89} \in$ $\mathcal{A}^{6}(11)$. Its associated Lehrer-Solomon graph $\gamma$ is
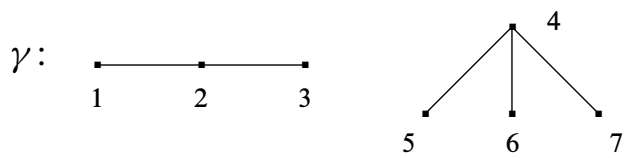

(b) Consider the monomial $\mu=x_{H_{*}} G_{I_{*} J_{*}} \in E_{6}^{*}(X, 11)$ given by

$$
x_{h_{1}} \otimes 1 \otimes 1 \otimes x_{h_{4}} \otimes 1 \otimes 1 \otimes 1 \otimes x_{h_{8}} \otimes 1 \otimes x_{h_{10}} \otimes x_{h_{11}} G_{12} G_{23} G_{45} G_{46} G_{47} G_{89} .
$$

Its associated marked graph $\Gamma$ is
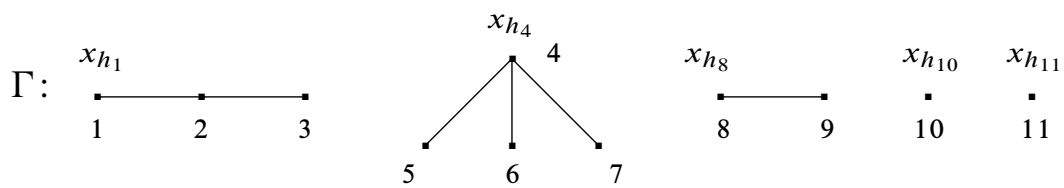

Conversely, we associate to a given marked (simple) graph $\Gamma$ an element in $E_{*}^{*}(X, n)$ : if there is no edge and the marks of the vertices $1,2, \ldots, n$ are $x_{h_{1}}, \ldots, x_{h_{n}}$, the corresponding element is $x_{h_{1}} \otimes \cdots \otimes x_{h_{n}} \in E_{0}^{*}$; otherwise we take the product, in the lexicographic order, of the exterior factors $G_{i j}$ corresponding to the edges $\{i, j\}$ and the scalar is the product of marks $x_{h_{1}} \otimes 1 \otimes \cdots \otimes x_{h_{i}} \otimes \cdots$, where the factor $x_{h_{i}}$ is the mark on the connected component having $i$ as the smallest element (all the other factors are equal to 1 ).

Due to the acyclicity of Lehrer-Solomon graphs (see Remark 2.2), we will consider only marked forests (all the connected components are trees). If we restrict the correspondence $\left\{\right.$ monomials in $\left.E_{*}^{*}(X, n)\right\} \rightarrow$ marked graphs\} to the canonical basis (Bezrukavnikov), we obtain only marked monotonic graphs. 
Definition 2.5 A tree with vertices $\left\{1 \leq i_{1}<i_{2}<\cdots<i_{p} \leq n\right\}$ is monotonic if, for any vertex $i_{k}$, the unique path from $i_{1}$ to $i_{k}$ is strictly increasing:

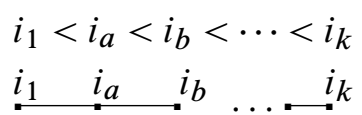

(choosing the root $i_{1}$, the rooted tree is monotonic). A forest with vertices $\{1,2, \ldots, n\}$ is monotonic if all its trees are monotonic.

Example 2.6 The tree

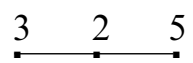

is monotonic, but

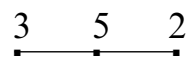

is not.

Remark 2.7 There is one to one correspondence

\{monomials in the canonical basis of $\left.E_{*}^{*}(X, n)\right\} \leftrightarrow$ marked monotonic forests\}.

Proof Let us suppose that the graph $\Gamma$ associated to a canonical monomial $G_{I_{*} J_{*}}$ $\left(j_{1}<j_{2}<\cdots<j_{q}, i_{a}<j_{a}\right.$ for $\left.a=1,2, \ldots, q\right)$ is connected; from its Euler characteristic we find that $\operatorname{card}\left(I_{*} \cup J_{*}\right)=q+1$. If $\Gamma$ is not monotonic, there is a path $i_{1}-\cdots-j-k-h$ such that $\left(i_{1} \leq\right) j<k, k>h$, and this corresponds to a forbidden product $G_{j k} G_{h k}$ in $G_{I_{*} J_{*}}$. Conversely, to any monotonic tree (or forest) corresponds a product $G_{I_{*} J_{*}}$ from the canonical basis: a vertex $j$, distinct from the minimal vertex $i$ in the same connected component, is joined with a unique vertex $h$, and this is smaller than $j$, namely the second last vertex on the path from $i$ to $j$; therefore $j$ appears only once on the second position, hence in the sequence $J_{*}$.

There is an obvious action of the symmetric group $\mathcal{S}_{n}$ on the set of marked graphs: the natural action of $\mathcal{S}_{n}$ on the set of vertices $\{1,2, \ldots, n\}$ induces an action on the set of edges and an action on the connected components and the corresponding marks. The set of monotonic marked forests is not $\mathcal{S}_{n}$-stable, like the set of monomials in the canonical basis of the Križ model. But $E_{*}^{*}(X, n)$ and the $\mathbb{Q}$ vector space which is generated by marked monotonic forests are $\mathcal{S}_{n}$-stable and these two vector spaces will be identified. 
In the next examples two $\mathcal{S}_{4}$-orbits in the $\mathbb{Q}$-span of monotonic marked forests are described: $\oplus$ and $\ominus$ stand for the sum and difference in this vector space and $\tau_{i}=(i, i+1)(i=1,2,3)$ are the Coxeter generators of $\mathcal{S}_{4}$. In order to save space, we have that the bullet $\bullet$ corresponds to the vertex 1 , the root of the tree, and the vertices connected to 1 are, from left to right, written in increasing order. Hence,

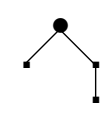

is the short form of:

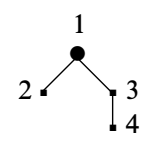

But the rooted tree

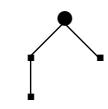

is ambiguous, and therefore we will write

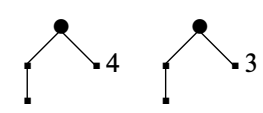

for the short form of

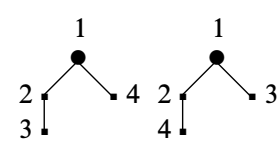

respectively. For the same reason the (unique) mark $x_{h_{1}} \in \mathcal{B}$ is omitted.

\section{Example 2.8}

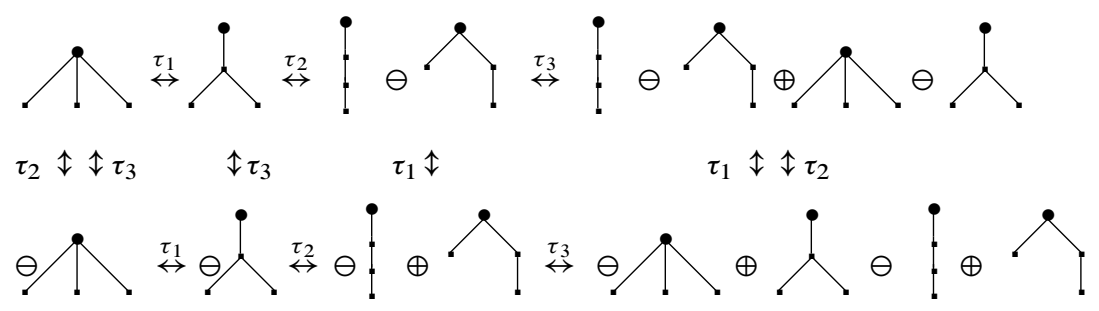




\section{Example 2.9}

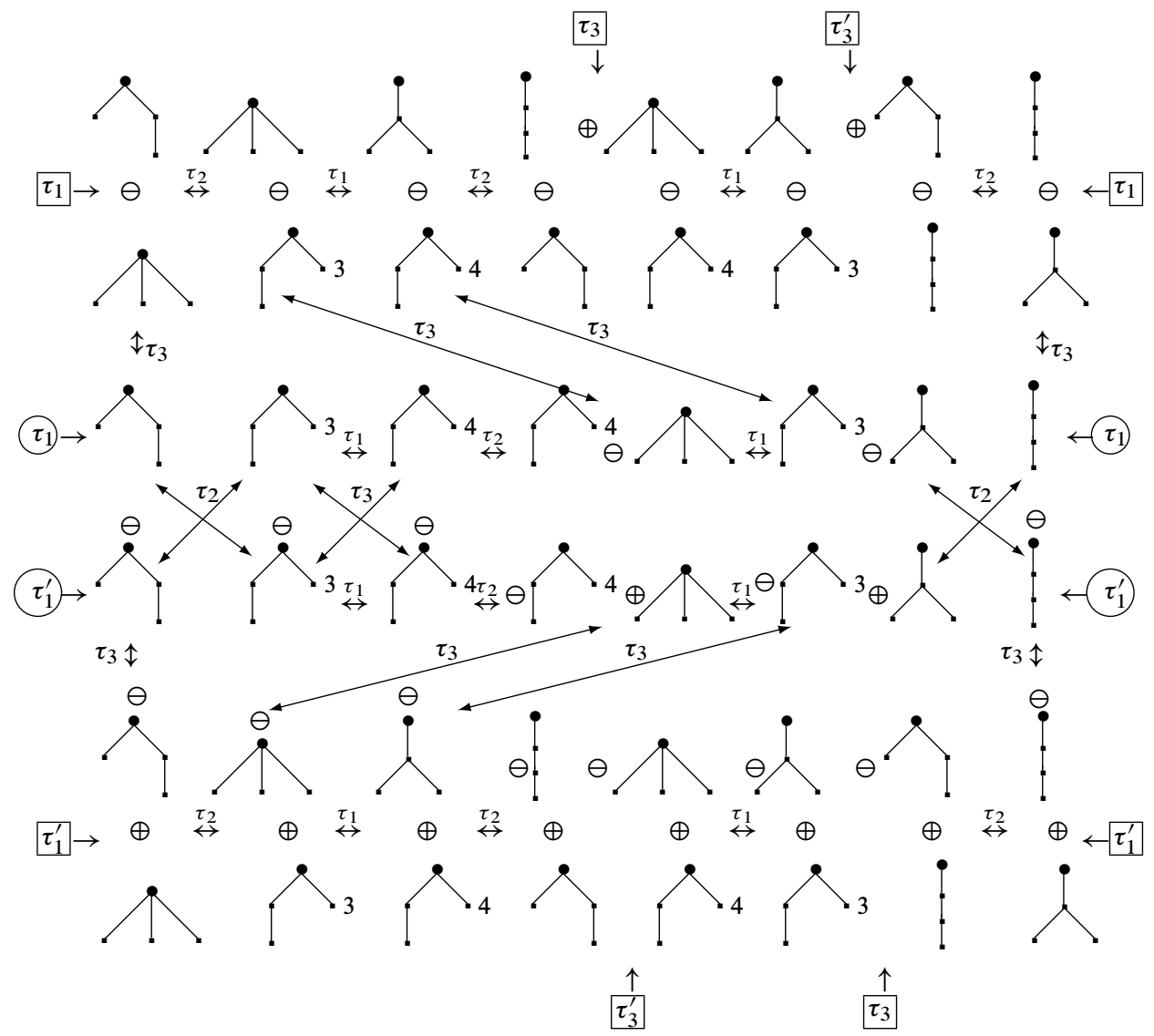

In the first example we have a "small" orbit: the dimension of the representation is 3 and it is isomorphic to $V(2,1,1)$. In the second one we see a "complete" orbit: the dimension is 6 and the representation is $V(3,1) \oplus V(2,1,1)$. The second example suggests the next definition.

Definition 2.10 We say that two monotonic marked forests are of the same type, $\left(\Gamma, H_{*}\right) \sim\left(\Gamma^{\prime}, H_{*}^{\prime}\right)$, if there is a permutation $\sigma \in \mathcal{S}_{n}$ which induces a bijection between the connected components of the two graphs, preserving the number of elements of the corresponding components and their marks.

It is clear that marked monotonic forests in the same $\mathcal{S}_{n}$-orbit are of the same type, but not conversely. A complete system of representatives for this equivalence relation is given by forests of bamboos: 


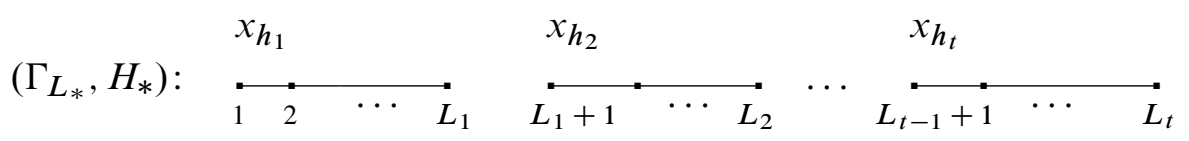

where the sequence of lengths of the bamboos $L_{*}=\left(\lambda_{1}, \lambda_{2}, \ldots, \lambda_{t}\right)$ is decreasing $\lambda_{1} \geq \lambda_{2} \geq \cdots \geq \lambda_{t}, L_{i}=\lambda_{1}+\lambda_{2}+\cdots+\lambda_{i}, L_{t}=n$, and, for equal lengths, the marks are in a decreasing order. We split the $\mathcal{S}_{n}$-modules $E_{q}^{k}(X, n)$ into smaller pieces using the type of the associated monotonic marked forests.

Definition 2.11 For a given marked forest of bamboos $\left(\Gamma_{L_{*}}, H_{*}\right)$ we define the subspace of type $\left(L_{*}, H_{*}\right)$ as the linear span of monomials with the associated marked graph of type $\left(\Gamma_{L_{*}}, H_{*}\right)$; this will be denoted by $E_{*}^{*}\left(L_{*}, H_{*}\right)$.

Example 2.12 For the type $\left(\Gamma_{(3,1,1)},\left(h_{1}, h_{2}, h_{3}\right)\right)$, where $x_{h_{2}} \succ x_{h_{3}}$,

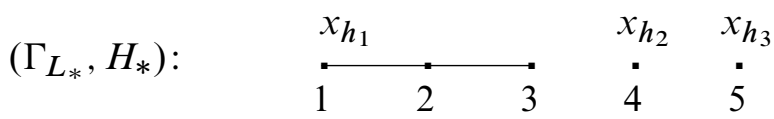

the associated space $E_{*}^{*}\left(L_{*}, H_{*}\right)$ is of dimension 40 and its canonical basis is given by the monomials

$$
\begin{array}{cc}
x_{h_{1}} \otimes 1 \otimes 1 \otimes x_{h_{2}} \otimes x_{h_{3}} G_{12} G_{13}, & x_{h_{1}} \otimes 1 \otimes 1 \otimes x_{h_{3}} \otimes x_{h_{2}} G_{12} G_{13}, \\
x_{h_{1}} \otimes 1 \otimes 1 \otimes x_{h_{2}} \otimes x_{h_{3}} G_{12} G_{23}, & x_{h_{1}} \otimes 1 \otimes 1 \otimes x_{h_{3}} \otimes x_{h_{2}} G_{12} G_{23}, \\
\ldots & \ldots \\
x_{h_{2}} \otimes x_{h_{3}} \otimes x_{h_{1}} \otimes 1 \otimes 1 G_{34} G_{45}, & x_{h_{3}} \otimes x_{h_{2}} \otimes x_{h_{1}} \otimes 1 \otimes 1 G_{34} G_{45} .
\end{array}
$$

If in this example $x_{h_{2}}=x_{h_{3}}$, the dimension of $E_{*}^{*}\left(L_{*}, H_{*}\right)$ is 20 .

To a given type $\left(L_{*}, H_{*}\right), L_{*}=\left(\lambda_{1}, \ldots, \lambda_{t}\right), H_{*}=\left(h_{1}, \ldots, h_{t}\right)$, we will associate two integers,

$$
\left|L_{*}\right|=\sum_{i=1}^{t}\left(\lambda_{i}-1\right), \quad\left|H_{*}\right|=\sum_{i=1}^{t} \operatorname{deg}\left(x_{h_{i}}\right)
$$

Theorem 2.13 The bihomogenous components $E_{q}^{k}(X, n)$ can be decomposed into a direct sum of monogenic $\mathcal{S}_{n}$-submodules

$$
E_{q}^{k}(X, n)=\bigoplus_{\substack{\left|L_{*}\right|=q \\\left|H_{*}\right|=k-q(2 m-1)}} E_{q}^{k}\left(L_{*}, H_{*}\right)
$$

In particular, we have that the multiplicities of the irreducible $\mathcal{S}_{n}$-submodules of each term $E_{q}^{k}\left(L_{*}, H_{*}\right)=\bigoplus_{\lambda \vdash n} m_{\lambda} V(\lambda)$ satisfy the relations $m_{\lambda} \leq \operatorname{dim} V(\lambda)$. 
Proof The $\mathcal{S}_{n}$-module $E_{q}^{k}(X, n)$ is the direct sum $\bigoplus E_{q}^{k}\left(L_{*}, H_{*}\right)$ by the very definition of monomials of type $\left(L_{*}, H_{*}\right)$. We will show that

(a) for any type $\left(L_{*}, H_{*}\right)$, the vector space $E\left(L_{*}, H_{*}\right)$ is $\mathcal{S}_{n}$-stable;

(b) a generator of the $\mathcal{S}_{n}$-module $E_{*}^{*}\left(L_{*}, H_{*}\right)$ is the monomial corresponding to the marked monotonic bamboo $\left(L_{*}, H_{*}\right)$ :

$$
\begin{aligned}
& \mu_{\left(L_{*}, H_{*}\right)} \\
& \quad=p_{1}^{*}\left(x_{h_{1}}\right) p_{L_{1}+1}^{*}\left(x_{h_{2}}\right) \cdots p_{L_{t-1}+1}^{*}\left(x_{h_{t}}\right) \overline{G_{1 L_{1}}} \cdot \overline{G_{L_{1}+1, L_{2}}} \cdots \overline{G_{L_{t-1}+1, L_{t}}}
\end{aligned}
$$

(here $\overline{G_{a b}}=G_{a, a+1} G_{a+1, a+2} \cdots G_{b-1, b}$ ).

The last claim of the theorem is a consequence of the $\mathcal{S}_{n}$ - equivariant surjection

$$
\mathbb{Q}\left[\mathcal{S}_{n}\right] \rightarrow E\left(L_{*}, H_{*}\right), \quad \sigma \mapsto \sigma \cdot \mu_{\left(L_{*}, H_{*}\right)} .
$$

To prove (a) it is enough to consider the action of the transpositions $\tau_{i}=(i, i+1)$ on the tree $T$ containing the vertices $i, i+1$ or on the disjoint union of two trees, $T^{\prime}$ and $T^{\prime \prime}$, containing $i$ and $i+1$ respectively. In the case of one tree, the transform $\tau_{i} T$ is again a monotonic tree (and $\tau_{i} T \sim T$ ) if the path from 1 to $i+1$ does not contain $i$. Otherwise the monotonic path $1-\cdots-h-i-(i+1)$ is transformed into $1-\cdots-h-(i+1)-i$ and the corresponding factor $G_{h, i+1} G_{i, i+1}$ should be replaced by $G_{h, i} G_{i, i+1}-G_{h, i} G_{h, i+1}$. The resulting monomials have monotonic trees of the same type with $T$. The case of two trees, $i$ in $T^{\prime}$ and $i+1$ in $T^{\prime \prime}$, is simpler: $\tau_{i}\left(T^{\prime} \sqcup T^{\prime \prime}\right)$ is a union of two monotonic trees $(h<i<j$ is equivalent to $h<i+1<j)$ and obviously this union is of the same type with $T^{\prime} \sqcup T^{\prime \prime}$.

It is enough to prove (b) for a monotonic tree: we will show by induction on $n$ that the bamboo $B_{n}=\mu_{\left(L_{*}=(n), H_{*}=\left(x_{h}\right)\right)}$ generate the module $\operatorname{Res}_{\mathcal{S}_{n-1}} E_{*}^{*}(X, n)$. If $\sigma$ is a permutation in $\mathcal{S}_{n-1}$, we denote by $\widetilde{\sigma}$ its extension to $\mathcal{S}_{n}: \widetilde{\sigma}(n)=n$. In the case $n=3$ there is a unique monotonic tree which is not the monotonic bamboo $B_{3}$, but this belongs to the $\mathcal{S}_{2}$-orbit of $B_{3}$ :

$$
2 \quad 13=\tau_{1}\left(\begin{array}{lll}
1 & 2 & 3
\end{array}\right)=\tau_{1}\left(B_{3}\right), \quad \tau_{1} \in \mathcal{S}_{2} .
$$

Let us consider a monotonic tree $T_{n}$ with $n$ vertices and its monotonic subtree $T_{n-1}=T_{n} \backslash\{n\}$ (by monotonicity, the vertex $n$ is connected to a unique other vertex, $h$ ). From the set of permutations $\pi \in \mathcal{S}_{n-1}$ with the property that $\pi\left(T_{n-1}\right)$ is still monotonic we choose one such that $\pi(h)=j$ is maximal. Now we start a second induction on $n-j$, the number of vertices of $\pi\left(T_{n}\right)$ lying on the branches starting from $j$. If this 
number is equal to 1 , then $j=n-1$ and, by induction on $n$ there are permutations $\sigma_{a}$ in $\mathcal{S}_{n-2}$ and constants $c_{a} \in \mathbb{Q}$ such that

$$
\pi\left(T_{n-1}\right)=\sum_{a} c_{a} \sigma_{a}\left(B_{n-1}\right)
$$

The extension of a permutation $\tilde{\sigma}$ of a permutation in $\mathcal{S}_{n-2}$ does not change the edge $(n-1)-n$ and we find $\tilde{\pi}\left(T_{n}\right)=\sum_{a} c_{a} \widetilde{\sigma}_{a}\left(B_{n}\right)$ and therefore

$$
T_{n}=\sum_{a} c_{a} \widetilde{\pi}^{-1} \sigma_{a}\left(B_{n}\right), \quad \text { where } \pi^{-1} \sigma_{a} \in \mathcal{S}_{n-1}
$$

If $j$ is less than $n-1$, then $j+1$ is connected to $j$ (by the maximality condition); applying the transposition $\tau_{j}$, we obtain a nonmonotonic tree $\tau_{j} \pi\left(T_{n}\right)$ (if $j \neq 1$ ). The sequence $k-(j+1)-j$ should be replaced: we obtain a difference of monotonic trees $T_{n}^{\prime}-T_{n}^{\prime \prime}$, in each of them $n$ is connected with $j+1$. The second induction will give two expansions

$$
T_{n}^{\prime}=\sum_{p} c_{p}^{\prime} \tilde{\sigma}_{p}^{\prime}\left(B_{n}\right), T_{n}^{\prime \prime}=\sum_{q} c_{q}^{\prime \prime} \tilde{\sigma}_{q}^{\prime \prime}\left(B_{n}\right)
$$

with $\sigma_{p}^{\prime}, \sigma_{q}^{\prime \prime} \in \mathcal{S}_{n-1}$. Therefore $T_{n}$ is in $\mathbb{Q}\left[\mathcal{S}_{n-1}\right]\left(B_{n}\right)$ :

$$
T_{n}=\sum_{p} c_{p}^{\prime} \overline{\pi^{-1} \tau_{j} \sigma_{p}^{\prime}}\left(B_{n}\right)-\sum_{q} c_{q}^{\prime \prime} \overline{\pi^{-1} \tau_{j} \sigma_{q}^{\prime \prime}}\left(B_{n}\right)
$$

In the case $j=1$ the transposition $\tau_{1}$ transforms $\pi\left(T_{n}\right)$ into a monotonic tree where $n$ is connected with 2 and we can apply the second induction.

Corollary 2.14 The $\mathcal{S}_{n-1}$ orbit of the monomial $x_{h} \otimes 1 \otimes \cdots 1 G_{12} G_{23} \cdots G_{n-1, n}$ coincides with $E_{n-1}^{(n-1)(2 m-1)+\left|x_{h}\right|}(n, h)$ and

$$
\operatorname{Res}_{\mathcal{S}_{n-1}}^{\mathcal{S}_{n}} E_{n-1}^{(n-1)(2 m-1)+\left|x_{h}\right|}(n, h) \cong \mathbb{Q}\left[\mathcal{S}_{n-1}\right]
$$

Proof In the proof of the theorem we obtained a surjective $\mathcal{S}_{n-1}$-map

$$
\mathbb{Q}\left[\mathcal{S}_{n-1}\right] \rightarrow \operatorname{Res}_{\mathcal{S}_{n-1}}^{\mathcal{S}_{n}} E_{n-1}^{(n-1)(2 m-1)+\left|x_{h}\right|}(n, h), \quad \sigma \mapsto \sigma \cdot B_{n}
$$

On the other hand the number of monotonic trees with $n$ vertices is $(n-1)$ !. 


\section{The $\mathcal{S}_{n}$-module $E_{q}^{k}\left(L_{*}, H_{*}\right)$}

In this section we will study the symmetric structure of the modules $E_{q}^{k}\left(L_{*}, H_{*}\right)$ using two methods. We extend the main result of Lehrer and Solomon [19] and we will give a general formula, but in an implicit form; next, in some particular cases, we will present explicit computations of the irreducible components and their multiplicities, using direct methods.

The symmetric structure of the bottom horizontal line is completely elementary: the types are given by $L_{*}=\left(1^{(n)}\right), H_{*}=\left(h_{1}^{\left(m_{1}\right)}, h_{2}^{\left(m_{2}\right)}, \ldots, h_{t}^{\left(m_{t}\right)}\right)$ :

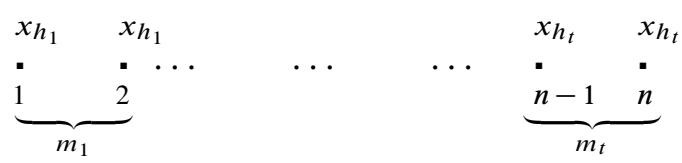

(here $m_{1}, m_{2}, \ldots, m_{t}$ are multiplicities of the elements $x_{h_{1}} \succ x_{h_{2}} \succ \cdots \succ x_{h_{t}}$ in $\mathcal{B}$ and $\left.m_{1}+\cdots+m_{t}=n\right)$.

Example 3.1 In the case of distinct marks (all the multiplicities $m_{i}$ are equal to 1) we obtain the largest possible "type" submodule

$$
E_{0}^{\left|H_{*}\right|}\left(1^{(n)},\left(h_{1}, h_{2}, \ldots, h_{n}\right)\right) \cong \mathbb{Q}\left[\mathcal{S}_{n}\right] .
$$

Proof As $\sigma^{-1}\left(x_{1} \otimes \cdots \otimes x_{n}\right)= \pm x_{\sigma(1)} \otimes \cdots \otimes x_{\sigma(n)}$, the character of this module is given by

$$
\chi_{E_{0}^{|H *|}}(\sigma)= \begin{cases}n ! & \sigma=\mathrm{id} \\ 0 & \sigma \neq \mathrm{id}\end{cases}
$$

This completes the proof.

Example 3.2 In the case of a unique mark $\left(m_{1}=n\right)$

$$
E_{0}^{n\left|x_{h}\right|}\left(1^{(n)}, h^{(n)}\right) \cong \begin{cases}V(n) & \text { if }\left|x_{h}\right| \text { is even or } n=1 \\ V(1,1, \ldots, 1) & \text { if }\left|x_{h}\right| \text { is odd and } n \geq 2\end{cases}
$$

Proof This is the consequence of the relation

$$
\tau_{i}\left(x_{h} \otimes \cdots \otimes x_{h}\right)=(-1)^{\left|x_{h}\right|}\left(x_{h} \otimes \cdots \otimes x_{h}\right) .
$$


For the general type corresponding to the discrete graph we will use the notation

$$
V^{\epsilon(m, h)}= \begin{cases}V(m) & \text { if } m=1 \text { or }\left|x_{h}\right| \text { is even } \\ V(1,1, \ldots, 1) & \text { if } m \geq 2 \text { and }\left|x_{h}\right| \text { is odd }\end{cases}
$$

Proposition 3.1 The $\mathcal{S}_{n}$ structure of the type $\left(L_{*}, H_{*}\right)$, where $L_{*}=\left(1^{(n)}\right), H_{*}=$ $\left(h_{1}^{\left(m_{1}\right)}, h_{2}^{\left(m_{2}\right)}, \ldots, h_{t}^{\left(m_{t}\right)}\right)$ is given by

$$
E_{0}^{\left|H_{*}\right|}\left(1^{(n)}, H_{*}\right) \cong \operatorname{Ind}_{\mathcal{S}_{m_{1}} \times \mathcal{S}_{m_{2}} \times \cdots \times \mathcal{S}_{m_{t}}} V^{\epsilon\left(m_{1}, h_{1}\right)} \otimes V^{\epsilon\left(m_{2}, h_{2}\right)} \otimes \cdots \otimes V^{\epsilon\left(m_{t}, h_{t}\right)} .
$$

Proof The symmetric group $\mathcal{S}_{n}$ acts transitively on the set of 1-dimensional spaces $\mathbb{Q}\left\langle x_{1} \otimes \cdots \otimes x_{n}\right\rangle$, where $m_{1}$ factors (on different positions) coincide with $x_{h_{1}}, \ldots, m_{t}$ factors coincide with $x_{h_{t}}$. The subgroup leaving invariant the 1-dimensional subspace

$$
\underbrace{x_{h_{1}} \otimes \cdots \otimes x_{h_{1}}}_{m_{1}} \otimes \cdots \otimes \underbrace{x_{h_{t}} \otimes \cdots \otimes x_{h_{t}}}_{m_{t}}
$$

is the direct product $\mathcal{S}_{m_{1}} \times \mathcal{S}_{m_{2}} \times \cdots \times \mathcal{S}_{m_{t}}$ (with the obvious notation: $\mathcal{S}_{m_{1}}$ acts on the subset $\left\{1,2, \ldots, m_{1}\right\}, \mathcal{S}_{m_{2}}$ acts on the subset $\left\{m_{1}+1, m_{1}+2, \ldots, m_{1}+m_{2}\right\}$, and so on) and the corresponding representation of this subgroup is $V^{\epsilon\left(m_{1}, h_{1}\right)} \otimes V^{\epsilon\left(m_{2}, h_{2}\right)} \otimes$ $\cdots \otimes V^{\epsilon\left(m_{t}, h_{t}\right)}$. General facts from the theory of induced representations (see for instance Serre [21, Chapter 7]) imply the result.

On the next horizontal line the types are given by a unique graph:

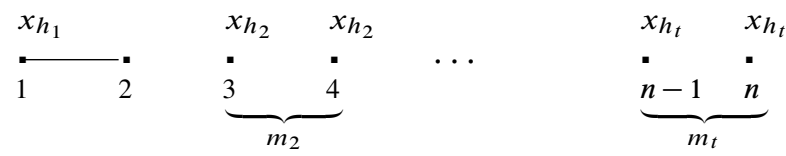

(here $x_{h_{2}} \succ \cdots \succ x_{h_{t}}$, but $h_{1}$ could be equal to one of $h_{2}, \ldots, h_{t}$ and we have $\left.2+m_{2}+\cdots+m_{t}=n\right)$.

Proposition 3.2 The $\mathcal{S}_{n}$ structure corresponding to the type $H_{*}=\left(h_{1}, h_{2}^{\left(m_{2}\right)}, \ldots, h_{t}^{\left(m_{t}\right)}\right)$, $L_{*}=\left(2,1^{(n-2)}\right)$, is given by

$$
E_{1}^{2 m-1+\left|H_{*}\right|}\left(L_{*}, H_{*}\right) \cong \operatorname{Ind}_{\mathcal{S}_{2} \times \mathcal{S}_{m_{2}} \times \cdots \times \mathcal{S}_{m_{t}}}^{\mathcal{S}_{n}} V(2) \otimes V^{\epsilon\left(m_{2}, h_{2}\right)} \otimes \cdots \otimes V^{\epsilon\left(m_{t}, h_{t}\right)} .
$$

Proof This is similar to the previous proof: the module $E_{*}^{*}\left(L_{*}, H_{*}\right)$ is the direct sum of 1 -dimensional subspaces $\mathbb{Q}\left\langle x_{1} \otimes \cdots \otimes x_{n} G_{i j}\right\rangle$, where on the $i^{\text {th }}$ position lies $x_{h_{1}}$, on the $j^{\text {th }}$ position is 1 , and $x_{h_{2}}$ is lying on $m_{2}$ (arbitrary) positions, .., $x_{h_{t}}$ on $m_{t}$ positions, and these subspaces are permuted by $\mathcal{S}_{n}$. The subgroup leaving invariant the line

$$
\mathbb{Q}\langle x_{h_{1}} \otimes 1 \otimes \underbrace{x_{h_{2}} \otimes x_{h_{2}} \otimes \cdots}_{m_{2}} \otimes \cdots \otimes \underbrace{\cdots \otimes x_{h_{t}}}_{m_{t}} G_{12}\rangle
$$


is the product $\mathcal{S}_{2} \times \mathcal{S}_{m_{2}} \times \cdots \times \mathcal{S}_{m_{t}}$ and the representation on this line is equivalent with $V(2) \otimes V^{\epsilon\left(m_{2}, h_{2}\right)} \otimes \cdots \otimes V^{\epsilon\left(m_{t}, h_{t}\right)}$, hence the result.

The following types appear on the third horizontal line:

(a) $\quad L_{*}=\left(3,1^{(n-3)}\right), H_{*}=\left(h_{1}, h_{2}^{\left(m_{2}\right)}, \ldots, h_{t}^{\left(m_{t}\right)}\right)$

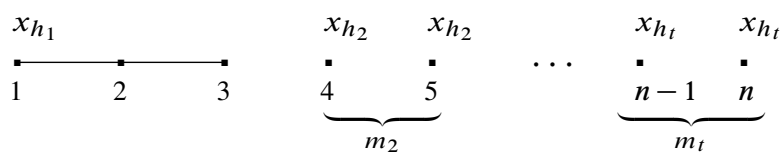

(b) $\quad L_{*}=\left(2^{(2)}, 1^{(n-4)}\right), H_{*}=\left(h_{1}^{(2)}, h_{2}^{\left(m_{2}\right)}, \ldots, h_{t}^{\left(m_{t}\right)}\right)$

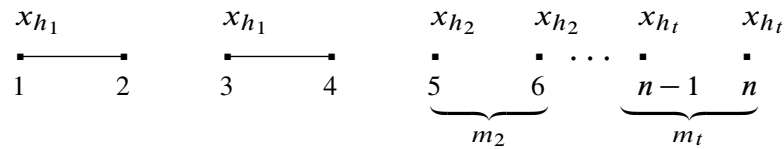

(c) $\quad L_{*}=\left(2^{(2)}, 1^{(n-4)}\right), H_{*}=\left(h_{1}, h_{2}, h_{3}^{\left(m_{3}\right)}, \ldots, h_{t}^{\left(m_{t}\right)}\right)$

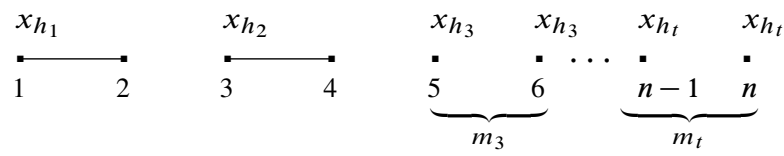

(In the first two cases $h_{2}, \ldots, h_{t}$ are distinct and $h_{1}$ could be one of them, in the third case $x_{h_{1}} \succ x_{h_{2}}, x_{h_{3}} \succ \cdots \succ x_{h_{t}}$ and $h_{1}$ or $h_{2}$ are not necessarily different from $\left.h_{3}, \ldots, h_{t}\right)$.

Example 3.3 For $n \geq 6$, the module $E_{2}^{2(2 m-1)}\left(L_{*}=\left(3,1^{(n-3)}\right), H_{*}=\left(1,1^{(n-3)}\right)\right)$ has the stable decomposition

$$
V(1)_{n} \oplus V(2)_{n} \oplus V(1,1)_{n} \oplus V(2,1)_{n} .
$$

Proof Computing directly the corresponding character, we find for an arbitrary permutation $\sigma \in \mathcal{S}_{n}$ of type $\left(i_{1} ; i_{2} ; \ldots ; i_{n}\right)$ (here $i_{q}$ is the number of cycles of length $q$ ): for any triple of $1-$ cycles $(i)(j)(k), 1 \leq i<j<k \leq n, \sigma$ fixes the monomials $G_{i j} G_{i k}$ and $G_{i j} G_{j k}$ and this gives $2\left(\begin{array}{c}i_{1} \\ 3\end{array}\right)$ such monomials. The 3 -cycle $(i, j, k)$ changes the sign of $G_{i j} G_{j k}$ and there is no other combination of cycles leaving $\mathbb{Q}\left\langle G_{i j} G_{j k}\right\rangle$ or $\mathbb{Q}\left\langle G_{i j} G_{i k}\right\rangle$ invariant. Therefore the character is

$$
\chi_{E_{2}^{2(2 m-1)}(\bullet-\bullet-\bullet \bullet \bullet)}\left(i_{1} ; \ldots ; i_{n}\right)=2\left(\begin{array}{c}
i_{1} \\
3
\end{array}\right)-i_{3} .
$$

Using Frobenius' formula we obtain the next results: 


\begin{tabular}{|c|c|}
\hline & $\chi_{V}\left(i_{1} ; \ldots ; i_{n}\right)$ \\
\hline$V(1)_{n}$ & $i_{1}-1$ \\
$V(1,1)_{n}$ & $\left(\begin{array}{c}i_{1}-1 \\
2\end{array}\right)-i_{2}$ \\
$V(2)_{n}$ & $\frac{1}{2} i_{1}\left(i_{1}-3\right)+i_{2}$ \\
$V(3)_{n}$ & $\frac{1}{6} i_{1}\left(i_{1}-1\right)\left(i_{1}-5\right)+i_{2}\left(i_{1}-1\right)+i_{3}$ \\
$V(2,1)_{n}$ & $\frac{1}{3} i_{1}\left(i_{1}-2\right)\left(i_{1}-4\right)-i_{3}$ \\
$V(3,1)_{n}$ & $\frac{1}{8} i_{1}\left(i_{1}-1\right)\left(i_{1}-3\right)\left(i_{1}-6\right)+i_{2}\left(\begin{array}{c}i_{1}-1 \\
2\end{array}\right)-\left(\begin{array}{c}i_{2} \\
2\end{array}\right)-i_{4}$ \\
\hline
\end{tabular}

As a consequence, we obtain the above decomposition.

In the unstable cases, $n=3,4,5$, this module decomposes as

$$
\begin{array}{ll}
n=3: & V(2,1), \\
n=4: & V(3,1) \oplus V(2,2) \oplus V(2,1,1), \\
n=5: & V(4,1) \oplus V(3,2) \oplus V(3,1,1) .
\end{array}
$$

The same decompositions appear if $H_{*}$ is replaced by $H_{*}=\left(h_{1}, h_{2}^{(n-3)}\right)$ with $\left|x_{h_{1}}\right|,\left|x_{h_{2}}\right|$ even.

Example 3.4 The module $E_{2}^{2(2 m-1)}\left(L_{*}=\left(2^{(2)}, 1^{(n-4)}\right), H_{*}=\left(1^{(2)} ; 1^{(n-4)}\right)\right)$, for $n \geq 7$, has the stable decomposition

$$
V(1)_{n} \oplus V(2)_{n} \oplus V(1,1)_{n} \oplus V(3)_{n} \oplus V(2,1)_{n} \oplus V(3,1)_{n} .
$$

Proof Direct computation of the character gives nonzero contributions only for factors of the form $(i)(j)(k)(l),(i)(j)(k, l),(i, j)(k, l)$ and $(i, j, k, l)$; the result is

$$
\chi_{E_{*}^{*}(\bullet \bullet \bullet-\bullet \bullet \bullet) ~}\left(i_{1} ; \ldots ; i_{n}\right)=3\left(\begin{array}{c}
i_{1} \\
4
\end{array}\right)+i_{2}\left(\begin{array}{c}
i_{1} \\
2
\end{array}\right)-\left(\begin{array}{c}
i_{2} \\
2
\end{array}\right)-i_{4}
$$

and from the same table we obtain the above decomposition.

In the unstable cases we obtain the decompositions

$$
\begin{array}{ll}
n=4: & V(3,1), \\
n=5: & V(4,1) \oplus V(3,2) \oplus V(3,1,1) \oplus V(2,2,1), \\
n=6: & V(5,1) \oplus V(4,2) \oplus V(4,1,1) \oplus V(3,3) \oplus V(3,2,1) .
\end{array}
$$

Example 3.5 In the case $n=4$ we have the decompositions

$$
E_{2}^{2(2 m-1)+2\left|x_{h}\right|}\left(\bullet_{1}^{x_{h}}-\bullet_{2} \bullet_{3}^{x_{h}}-\bullet_{4}\right) \cong \begin{cases}V(3,1) & \text { if }\left|x_{h}\right|=\text { even } \\ V(4) \oplus V(2,2) & \text { if }\left|x_{h}\right|=\text { odd }\end{cases}
$$


Proof The even case is the same as in the previous example; for the odd case direct computation of the character gives:

\begin{tabular}{|c|ccccc|}
\hline$\sigma$ & id & $(12)$ & $(123)$ & $(1234)$ & $(12)(34)$ \\
\hline$\chi(\sigma)$ & 3 & 1 & 0 & 1 & 3 \\
\hline
\end{tabular}

For instance,

$$
\begin{aligned}
(1234) p_{1}^{*}\left(x_{h}\right) p_{2}^{*}\left(x_{h}\right) G_{13} G_{24} & =p_{2}^{*}\left(x_{h}\right) p_{3}^{*}\left(x_{h}\right) G_{24} G_{13} \\
& =-p_{2}^{*}\left(x_{h}\right) p_{3}^{*}\left(x_{h}\right) G_{13} G_{24} \\
& =-p_{2}^{*}\left(x_{h}\right) p_{1}^{*}\left(x_{h}\right) G_{13} G_{24} \\
& =(-1)^{\left|x_{h}\right|+1} p_{1}^{*}\left(x_{h}\right) p_{2}^{*}\left(x_{h}\right) G_{13} G_{24} .
\end{aligned}
$$

This completes the proof.

Using some of these particular cases and the same proof as in Propositions 3.1 and 3.2, we obtain the following.

Proposition 3.3 The $\mathcal{S}_{n}$ structure of the modules corresponding to the types on the third horizontal line is given by:

(a) $E_{2}^{*}\left(L_{*}=\left(3,1^{(n-3)}\right), H_{*}=\left(h_{1}, h_{2}^{\left(m_{2}\right)}, \ldots, h_{t}^{\left(m_{t}\right)}\right)\right)$

$$
\cong \operatorname{Ind}_{\mathcal{S}_{3} \times \mathcal{S}_{m_{2}} \times \cdots \times \mathcal{S}_{m_{t}}}\left(V(2,1) \otimes V^{\epsilon\left(m_{2}, h_{2}\right)} \otimes \cdots \otimes V^{\epsilon\left(m_{t}, h_{t}\right)}\right)
$$

(b) $E_{2}^{*}\left(L_{*}=\left(2^{(2)}, 1^{(n-4)}\right), H_{*}=\left(h_{1}^{(2)}, h_{2}^{\left(m_{2}\right)}, \ldots, h_{t}^{\left(m_{t}\right)}\right)\right)$

$$
\cong \begin{cases}\operatorname{Ind}_{\mathcal{S}_{4} \times \mathcal{S}_{m_{2}} \times \cdots \times \mathcal{S}_{m_{t}}}\left(V(3,1) \otimes V^{\epsilon\left(m_{2}, h_{2}\right)} \otimes \cdots \otimes V^{\epsilon\left(m_{t}, h_{t}\right)}\right) & \left|x_{h_{1}}\right|=\text { even } \\ \operatorname{Ind}_{\mathcal{S}_{n} \times \mathcal{S}_{m_{2}} \times \cdots \times \mathcal{S}_{m_{t}}}^{\mathcal{S}_{n}}\left((V(4) \oplus V(2,2)) \otimes V^{\epsilon\left(m_{2}, h_{2}\right)} \otimes \cdots \otimes V^{\epsilon\left(m_{t}, h_{t}\right)}\right) & \left|x_{h_{1}}\right|=\text { odd }\end{cases}
$$

(c) $E_{2}^{*}\left(L_{*}=\left(2^{(2)}, 1^{(n-4)}\right), H_{*}=\left(h_{1}, h_{2}, h_{3}^{\left(m_{3}\right)}, \ldots, h_{t}^{\left(m_{t}\right)}\right)\right)$

$$
\begin{aligned}
& \cong \operatorname{Ind}_{\mathcal{S}_{2} \times \mathcal{S}_{2} \times \mathcal{S}_{m_{3}} \times \cdots \times \mathcal{S}_{m_{t}}}^{\mathcal{S}_{n}}\left(V(2) \otimes V(2) \otimes V^{\epsilon\left(m_{3}, h_{3}\right)} \otimes \cdots \otimes V^{\epsilon\left(m_{t}, h_{t}\right)}\right) \\
& \cong \operatorname{Ind}_{\mathcal{S}_{4} \times \mathcal{S}_{m_{3}} \times \cdots \times \mathcal{S}_{m_{t}}}\left((V(4) \oplus V(3,1) \oplus V(2,2)) \otimes V^{\epsilon\left(m_{3}, h_{3}\right)} \otimes \cdots \otimes V^{\epsilon\left(m_{t}, h_{t}\right)}\right)
\end{aligned}
$$

Now we will analyze the top horizontal line; we start with the upper-left vertex of the trapezoid, $E_{n-1}^{(n-1)(2 m-1)}(X, n)$. 
Example 3.6 For small values of $n$ the structure of this module is a consequence of the absence (for $n \geq 2$ ) of the one-dimensional submodules of $E_{n-1}^{*}(X, n)$ and of the

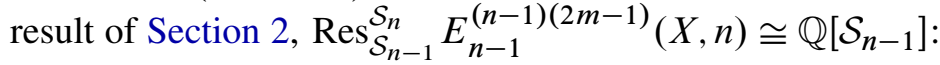

$$
\begin{array}{ll}
n=2: & E_{1}^{2 m-1} \cong V(2), \\
n=3: & E_{2}^{2(2 m-1)} \cong V(2,1), \\
n=4: & E_{3}^{3(2 m-1)} \cong V(3,1) \oplus V(2,1,1), \\
n=5: & E_{4}^{4(2 m-1)} \cong V(4,1) \oplus V(3,2) \oplus V(3,1,1) \oplus V(2,2,1) \oplus V(2,1,1,1) .
\end{array}
$$

By Poincaré duality, the right side of the trapezoid has the same structure.

Example 3.7 For $n=6$ there are seven $\mathcal{S}_{6}$-modules without one-dimensional submodules satisfying $\operatorname{Res}_{\mathcal{S}_{5}}^{\mathcal{S}_{6}} E_{5}^{5(2 m-1)} \cong \mathbb{Q}\left[\mathcal{S}_{5}\right]$. A direct combinatorial computation of the character of $E_{5}^{5(2 m-1)}$ will give its nonzero values:

\begin{tabular}{|c|cccc|}
\hline$\sigma$ & id & $(123456)$ & $(123)(456)$ & $(12)(34)(56)$ \\
$\chi(\sigma)$ & 120 & -1 & -3 & 8 \\
\hline
\end{tabular}

Therefore we get the next (asymmetric) decomposition:

$$
\begin{aligned}
& E_{5}^{5(2 m-1)} \cong \begin{array}{cccccc}
V(5,1) & \oplus & 2 V(4,2) & \oplus \quad V(4,1,1) & \oplus & 3 V(3,2,1)
\end{array} \\
& \oplus V(2,2,2) \oplus 2 V(3,1,1,1) \oplus V(2,2,1,1) \oplus V(2,1,1,1,1)
\end{aligned}
$$

One can find a character table of $\mathcal{S}_{6}$ in Ledermann [18].

Alternatively, we can use the next general result of Stanley [22] and Lehrer and Solomon [19] and the Frobenius reciprocity formula. Let us recall some notation necessary to present the Lehrer-Solomon theorem: consider the subgroup generated by the $n$-cycle $c_{n}=(1,2, \ldots, n),\left\langle c_{n}\right\rangle$, the elementary character $\varphi_{n}$ of this cyclic group, $\varphi_{n}\left(c_{n}\right)=e^{(2 \pi i) / n}$ and $\varepsilon_{n}$, the sign character of $\mathcal{S}_{n}$. More generally, for a partition $L_{*} \vdash n, L_{*}=\left(\lambda_{1} \geq \lambda_{2} \geq \cdots \lambda_{t} \geq 1\right)$, let us denote by $c_{L_{*}}$ the product of cycles $c_{\lambda_{1}} c_{\lambda_{2}} \cdots c_{\lambda_{t}}, c_{\lambda_{1}}=\left(1,2, \ldots, L_{1}\right), c_{\lambda_{2}}=\left(L_{1}+1, L_{1}+2, \ldots, L_{2}\right), \ldots$, $c_{\lambda_{t}}=\left(L_{t-1}+1, L_{t-1}+2, \ldots, L_{t}\right)$. Associated to $L_{*}$ we have the next diagram of groups and characters of one-dimensional representations: 


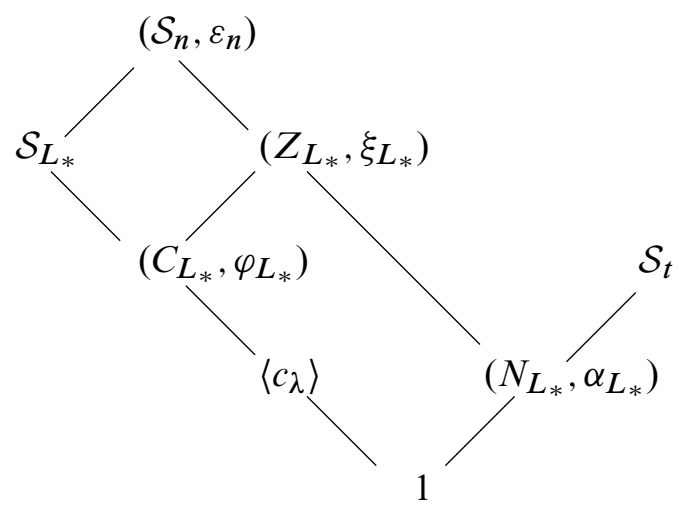

where $\left\langle c_{L_{*}}\right\rangle$ is the subgroup generated by $c_{L_{*}}, \mathcal{S}_{L_{*}}$ is the direct product

$$
\mathcal{S}_{L_{*}} \cong \mathcal{S}_{\lambda_{1}} \times \mathcal{S}_{\lambda_{2}} \times \cdots \times \mathcal{S}_{\lambda_{t}}
$$

and $C_{L_{*}}$ is the centralizer of $c_{L_{*}}$ in $\mathcal{S}_{L_{*}}$ :

$$
C_{L_{*}}=\left\langle c_{\lambda_{1}}\right\rangle \times\left\langle c_{\lambda_{2}}\right\rangle \times \cdots \times\left\langle c_{\lambda_{t}}\right\rangle
$$

The group $N_{L_{*}}$ is generated by the elements $v_{i}$, "block transpositions" corresponding to equal parts $\lambda_{i}=\lambda_{i+1}$ in the partition $L_{*}$ :

$v_{i}=\left(L_{i-1}+1, L_{i}+1\right)\left(L_{i-1}+2, L_{i}+2\right) \cdots\left(L_{i}=L_{i-1}+\lambda_{i}, L_{i+1}=L_{i}+\lambda_{i+1}\right)$

The last group, $Z_{L_{*}}$, is the centralizer of $c_{L_{*}}$ in $\mathcal{S}_{n}$ and it is a semidirect product

$$
Z_{L_{*}}=C_{L_{*}} \rtimes N_{L_{*}} .
$$

Lehrer and Solomon's main formula involves the next characters:

$$
\begin{aligned}
\varphi_{L_{*}}: C_{L_{*}} \rightarrow \mathbb{C}^{*}, & \varphi_{L_{*}}=\left.\left(\varphi_{\lambda_{1}} \otimes \cdots \otimes \varphi_{\lambda_{t}}\right) \cdot \varepsilon_{n}\right|_{C_{L *}} \\
\alpha_{L_{*}}: N_{L_{*}} \rightarrow \mathbb{C}^{*}, & \alpha_{L_{*}}\left(v_{i}\right)=(-1)^{\lambda_{i}+1} \\
\xi_{L_{*}}: Z_{L_{*}} \rightarrow \mathbb{C}^{*}, & \xi_{L_{*}}=\alpha_{L_{*}} \cdot \varphi_{L_{*}}
\end{aligned}
$$

Theorem 3.8 (Stanley [22], Lehrer and Solomon [19]) The representation of $\mathcal{S}_{n}$ on the top component $\mathcal{A}^{n-1}(n)$ of the Arnold algebra has the character

$$
\chi_{\mathcal{A}^{n-1}(n)}=\varepsilon_{n} \operatorname{Ind}_{\left\langle c_{n}\right\rangle}^{\mathcal{S}_{n}}\left(\varphi_{n}\right) .
$$


On the upper horizontal line the types are parameterized by the $n$-monotonic bamboo and the monomials from the fixed basis $\mathcal{B}$ :

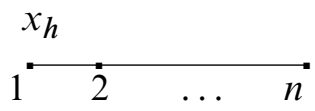

Proposition 3.4 The $\mathcal{S}_{n}$ structure of the top component is given by

$$
\begin{aligned}
\chi_{E_{n-1}^{(n-1)(2 m-1)+\left|x_{h}\right|}(n, h)} \cong \varepsilon_{n} \operatorname{Ind}_{\left\langle c_{n}\right\rangle}^{\mathcal{S}_{n}}\left(\varphi_{n}\right), \\
\chi_{E_{n-1}^{(n-1)(2 m-1)+i}(X, n)} \cong \beta_{i} \cdot \varepsilon_{n} \operatorname{Ind}_{\left\langle c_{n}\right\rangle}^{\mathcal{S}_{n}}\left(\varphi_{n}\right),
\end{aligned}
$$

where $\beta_{i}$ is the $i^{\text {th }}$ Betti number.

Proof As the action of the symmetric group does not change the coefficients

$$
\sigma\left(x_{h} \otimes 1 \otimes \cdots \otimes 1 G_{I_{*} J_{*}}\right)=p_{\sigma(1)}^{*}\left(x_{h}\right) \cdot \sigma\left(G_{I_{*} J_{*}}\right)=p_{1}^{*}\left(x_{h}\right) \cdot \sigma\left(G_{I_{*} J_{*}}\right),
$$

we have the $\mathcal{S}_{n}$-decomposition

$$
E_{n-1}^{(n-1)(2 m-1)+i}(X, n) \cong \bigoplus_{x_{h} \in \mathcal{B} \cap H^{i}(X)} p_{1}^{*}\left(x_{h}\right) \cdot \mathcal{A}^{n-1}(n) .
$$

Example 3.9 If the number of points is an odd prime number $p$, then the multiplicities $m_{\lambda}$ of the irreducible $\mathcal{S}_{p}$-modules are given by

$$
\begin{gathered}
E_{p-1}^{(p-1)(2 m-1)+\left|x_{h}\right|}(p, h) \cong \bigoplus_{\lambda \vdash p} m_{\lambda} V(\lambda), \\
m_{\lambda}=\frac{1}{p}\left(\operatorname{dim} V(\lambda)-\chi_{\lambda}\left(c_{p}\right)\right) .
\end{gathered}
$$

Proof From the Frobenius reciprocity formula we obtain the expansion (here $V\left(\lambda^{\varepsilon}\right)=$ $V(1,1, \ldots, 1) \otimes V(\lambda)$ with character $\left.\chi_{\lambda \varepsilon}\right)$

$$
\begin{aligned}
m_{\lambda}=\left\langle\chi_{V}, \varepsilon_{p} \operatorname{Ind}_{\left\langle c_{p}\right\rangle}^{\mathcal{S}_{p}}\left(\varphi_{p}\right)\right\rangle_{\mathcal{S}_{p}} & =\left\langle\chi_{\lambda \varepsilon}, \operatorname{Ind}_{\left\langle c_{p}\right\rangle}^{\mathcal{S}_{p}}\left(\varphi_{p}\right)\right\rangle_{\mathcal{S}_{p}} \\
& =\left\langle\operatorname{Res}_{\left\langle c_{p}\right\rangle}^{\mathcal{S}_{p}} \chi_{\lambda \varepsilon},\left(\varphi_{p}\right)\right\rangle_{\mathbb{Z}_{p}}=\frac{1}{p} \sum_{k=0}^{p-1} \chi_{\lambda \varepsilon}\left(c_{p}^{k}\right) e^{(2 k \pi i) / p}
\end{aligned}
$$

in which all the values $\chi_{\lambda \varepsilon}\left(c_{p}^{k}\right)$ are equal but not the first one:

$$
\chi_{\lambda \varepsilon}\left(c_{p}^{0}\right)=\operatorname{dim} V\left(\lambda^{\varepsilon}\right)=\operatorname{dim} V(\lambda)
$$

Now we consider the type $L_{*}=\left(\lambda_{1}, \lambda_{2}, \ldots, \lambda_{t}\right), H_{*}=\left(1^{(t)}\right)\left(\left|L_{*}\right|=\sum\left(\lambda_{i}-1\right)\right)$. Translated into the language of types, the main formula of Lehrer and Solomon is the following. 
Theorem 3.10 (Lehrer and Solomon [19]) The representation of $\mathcal{S}_{n}$ on the component $E_{\left|L_{*}\right|}^{\left|L_{*}\right|(2 m-1)}\left(L_{*}, 1^{(t)}\right)$ has the character

$$
\chi_{E_{|L *|}^{|L *|(2 m-1)}\left(L_{*}, 1^{(t)}\right)}=\operatorname{Ind}_{Z_{L *}}^{\mathcal{S}_{n}}\left(\xi_{L_{*}}\right) .
$$

To extend this to a general type $L_{*}=\left(\lambda_{1}, \ldots, \lambda_{t}\right), H_{*}=\left(h_{1}, h_{2}, \ldots, h_{t}\right)$, we modify the notation of Lehrer and Solomon as follows: the group $N_{\left(L_{*}, H_{*}\right)}$ is generated by the elements $v_{i}$ corresponding to the transposition of equal marked bamboos: $\lambda_{i}=\lambda_{i+1}$, $h_{i}=h_{i+1}$ (remember that for equal lengths $\lambda_{c}=\lambda_{c+1}=\cdots=\lambda_{d}$, the corresponding marks are decreasing, not necessarily strictly: $x_{h_{c}} \succeq x_{h_{c+1}} \succeq \cdots \succeq x_{h_{d}}$ ); of course, $v_{i}$ are given by the same product of disjoint transpositions.

The subgroup $Z_{\left(L_{*}, H_{*}\right)}$ is defined by the same formula,

$$
Z_{\left(L_{*}, H_{*}\right)}=C_{L_{*}} \rtimes N_{\left(L_{*}, H_{*}\right)},
$$

but now is, in general, smaller than the centralizer of $c_{L_{*}}$ in $\mathcal{S}_{n}$. The character $\varphi_{L_{*}}: C_{L_{*}} \rightarrow \mathbb{C}^{*}$ is given by the same formula: for a permutation $\sigma \in \mathcal{S}_{L_{*}}=$ $\mathcal{S}_{l_{1}} \times \mathcal{S}_{l_{2}} \times \cdots \times \mathcal{S}_{l_{t}}$, the sign of $\sigma\left(x_{h_{1}} \otimes 1 \otimes \cdots \otimes x_{h_{2}} \otimes 1 \cdots G_{I_{*} J_{*}}\right)$ is given only by the permutation of the exterior factors $G_{i j}$, like in Lehrer and Solomon definition of $\varphi_{L_{*}}$. The coefficients $\cdots \otimes x_{h_{i}} \otimes 1 \cdots \otimes x_{h_{i}} \otimes 1 \otimes \cdots$ do have a contribution to the sign after the action of a permutation $\rho \in N_{\left(L_{*}, H_{*}\right)}$ if the degree $\left|x_{h_{i}}\right|$ is odd; therefore the character $\alpha_{\left(L_{*}, H_{*}\right)}$ should be modified as follows:

$$
\alpha_{\left(L_{*}, H_{*}\right)}= \begin{cases}(-1)^{\lambda_{i}+1} & \text { if }\left|x_{h_{i}}\right| \text { is even, } \\ (-1)^{\lambda_{i}} & \text { if }\left|x_{h_{i}}\right| \text { is odd, }\end{cases}
$$

and accordingly the character $\xi$ is modified:

$$
\xi_{\left(L_{*}, H_{*}\right)}=\varphi_{L_{*}} \cdot \alpha_{\left(L_{*}, H_{*}\right)} \cdot
$$

Finally we obtain the character of the Križ algebra $E_{*}^{*}(X, n)$.

Theorem 3.11 $\quad$ (a) The $\mathcal{S}_{n}$ representation of the submodule $E_{*}^{*}\left(L_{*}, H_{*}\right)$ has the character

$$
\chi_{E_{*}^{*}\left(L_{*}, H_{*}\right)}=\operatorname{Ind}_{Z_{\left(L_{*}, H_{*}\right)}}^{\mathcal{S}_{n}}\left(\xi_{\left(L_{*}, H_{*}\right)}\right) .
$$

(b) The $\mathcal{S}_{n}$ representation of the component $E_{q}^{k}(X, n)$ has the character

$$
\chi_{E_{q}^{k}(X, n)}=\sum_{\substack{\left|L_{*}\right|=q \\\left|H_{*}\right|+q(2 m-1)=k}} \operatorname{Ind}_{Z_{\left(L_{*}, H_{*}\right)}}^{\mathcal{S}_{n}}\left(\xi_{\left(L_{*}, H_{*}\right)}\right) .
$$


(c) The $\mathcal{S}_{n}$ representation of the Križ algebra $E_{*}^{*}(X, n)$ has the character

$$
\chi_{E_{*}^{*}(X, n)}=\sum_{\left(L_{*}, H_{*}\right)} \operatorname{Ind}_{Z_{\left(L_{*}, H_{*}\right)}}^{\mathcal{S}_{n}}\left(\xi_{\left(L_{*}, H_{*}\right)}\right) .
$$

Proof For a given partition $\Lambda_{*}=\left(\Lambda_{1}, \Lambda_{2}, \ldots, \Lambda_{t}\right)$ of the set $\{1,2, \ldots, n\}$, where $\left|\Lambda_{i}\right|=\lambda_{i}$, let us denote by $E_{*}^{*}\left(\Lambda_{*}, H_{*}\right)$ the span of monomials associated to the marked graphs having $\Lambda_{*}$ the set of connected components and $H_{*}$ the corresponding marks. The symmetric group $\mathcal{S}_{n}$ acts transitively on the components of the direct sum

$$
E_{*}^{*}\left(L_{*}, H_{*}\right)=\bigoplus_{\Lambda_{*}} E_{*}^{*}\left(\Lambda_{*}, H_{*}\right) .
$$

Now we fix the term $E_{*}^{*}\left(\Lambda_{*}, H_{*}\right)$ with

$$
\Lambda_{*}=\left(\left\{1, \ldots, L_{1}\right\},\left\{L_{1}+1, \ldots, L_{2}\right\}, \ldots,\left\{L_{t-1}+1, \ldots, L_{t}\right\}\right)
$$

and we follow the proof of Lehrer and Solomon [19, Section 4]: the subgroup of $\mathcal{S}_{n}$ leaving $E_{*}^{*}\left(\Lambda_{*}, H_{*}\right)$ invariant is $Z_{\left(L_{*}, H_{*}\right)}=C_{L_{*}} \rtimes N_{\left(L_{*}, H_{*}\right)}$ (from the set $v_{i}$ of permutations of connected components of equal size we have to consider only the permutations of components with the same mark). The action of this subgroup on $E_{*}^{*}\left(\Lambda_{*}, H_{*}\right)$ is a composition of

(C) the action of $C_{L_{*}}$ on each component separately, and this is given componentwise by Proposition 3.4; its character is $\varphi_{L_{*}}$;

(N) the action of $N_{\left(L_{*}, H_{*}\right)}$ which permutes identical marked trees; the rules of changing the sign were already explained.

The last two formulae of the theorem are direct consequences of the first one.

\section{Proofs of Propositions 1.1 and 1.2}

In this section we will give a proof of Propositions 1.1 and 1.2. From the last section in [2] we will use the $\mathcal{S}_{n}$-decomposition of $E_{1}^{2 m-1}(X, n) \cong \mathcal{A}^{1}(n)$ and also the bases for the irreducible $\mathcal{S}_{n}$-submodules described in this paper.

Proposition 4.1 [2] The structure of the $\mathcal{S}_{n}$-module $E_{1}^{2 m-1}(X, n)$ is given by

$$
\begin{aligned}
& E_{1}^{2 m-1}(X, 2) \cong V(2), \\
& E_{1}^{2 m-1}(X, 3) \cong V(3) \oplus V(2,1), \\
& E_{1}^{2 m-1}(X, n) \cong V(n) \oplus V(n-1,1) \oplus V(n-2,2) \text { for } n \geq 4 .
\end{aligned}
$$


We choose one nonzero element from each $\mathcal{S}_{n}$-submodule,

$$
\begin{aligned}
G^{n} & =\sum_{i<j} G_{i j} \quad \text { in } V(n), \\
G_{12}^{n} & =\sum_{k \geq 3}\left(G_{1 k}-G_{2 k}\right) \quad \text { in } V(n-1,1), \\
G_{1234} & =G_{14}-G_{13}+G_{23}-G_{24} \quad \text { in } V(n-2,2),
\end{aligned}
$$

and by direct computation we find nonzero differentials.

Lemma 4.1 The images of the elements $G^{n}$ and $G_{12}^{n}$ under the composition

$$
E_{1}^{2 m-1} \stackrel{d}{\longrightarrow} E_{0}^{2 m} \stackrel{p r}{\longrightarrow} \bigoplus_{i=1}^{n} p_{i}^{*}\left(H^{2 m}\right)
$$

are given by

$$
\begin{aligned}
G^{n} & \mapsto(n-1) \sum_{i=1}^{n} p_{i}^{*}(w), \\
G_{12}^{n} & \mapsto(n-2) p_{1}^{*}(w)-(n-2) p_{2}^{*}(w) .
\end{aligned}
$$

Lemma 4.2 Let $x$ and $y$ be cohomology classes of positive degree ( $x$ in $\mathcal{B}$ and $y$ in the dual bases $\mathcal{B}^{*}$ ) such that $x y=w$. The image of the element $G_{1234}$ under the composition

$$
E_{1}^{2 m-1} \stackrel{d}{\longrightarrow} E_{0}^{2 m} \stackrel{p r}{\longrightarrow} \bigoplus_{i<j} p_{i}^{*}(\mathbb{Q}(x)) p_{j}^{*}(\mathbb{Q}(y))
$$

is given by

$$
G_{1234} \mapsto p_{1}^{*}(x) p_{4}^{*}(y)-p_{1}^{*}(x) p_{3}^{*}(y)+p_{2}^{*}(x) p_{3}^{*}(y)-p_{2}^{*}(x) p_{4}^{*}(y) .
$$

Proposition 4.2 If $X$ is a smooth complex projective variety $\left(X \neq \mathbb{C} P^{1}\right)$, then the "first" differential is injective:

$$
d: E_{1}^{2 m-1}(X, n) \succ E_{0}^{2 m}(X, n)
$$

Proof Using twice the Schur lemma for the $\mathcal{S}_{n}$-morphisms

$$
V(n), V(n-1,1) \hookrightarrow E_{1}^{2 m-1}(X, n) \stackrel{d}{\longrightarrow} E_{0}^{2 m}(X, n),
$$

these two submodules have isomorphic images through $d$ in $E_{0}^{2 m}$ and trivial kernels because these morphisms are nonzero: $d\left(G^{n}\right) \neq 0, d\left(G_{12}^{n}\right) \neq 0$. If $X$ is of complex 
dimension $m$ greater than two, we can take in Lemma $4.2 x$ to be the Kähler class and $y=x^{m-1}$; if $X$ is a smooth projective curve, but not the projective line, the equation $x y=w$ has also nontrivial solutions.

Remark 4.3 In the remaining case, the differential

$$
d: E_{1}^{1}\left(\mathbb{C} P^{1}, n\right) \rightarrow E_{0}^{2}\left(\mathbb{C} P^{1}, n\right)
$$

is injective only for $n=2,3$; for $n \geq 4$ we obtain

$$
H_{1}^{1}\left(F\left(\mathbb{C} P^{1}, n\right)\right) \cong V(2)_{n}
$$

We will see in the last section that in the case of $\mathbb{C} P^{1}, n \geq 4$, the differential $d: E_{q}^{q}\left(\mathbb{C} P^{1}\right) \rightarrow E_{q-1}^{q+1}\left(\mathbb{C} P^{1}\right)$ has a nontrivial kernel for $q=1,2, \ldots, n-3$ and it is injective for $q=n-2, n-1$.

Remark 4.4 As a consequence of [17], Propositions 4.2 and 1.1 are true for any formal space $X$ whose rational cohomology satisfies Poincaré duality but not for cohomology spheres (for $n \geq 4$ ).

Now we can give a proof of Proposition 1.1 by induction on $q$.

Proof of Proposition 1.1 Let us suppose that the differential

$$
d: E_{q-1}^{(q-1)(2 m-1)} \rightarrow E_{q-2}^{(q-1)(2 m-1)+1}
$$

is injective and let $u \in E_{q}^{q(2 m-1)}$ be a nonzero cocycle. Let $G_{i j}$ be the smallest exterior generator (in the reverse lexicographic order $G_{12}<G_{13}<G_{23}<G_{14}<\cdots<G_{n-1, n}$ ) which appears in a nonzero monomial (of the canonical basis) in $u: u=G_{i j} y+z$, $y \in E_{q-1}\left(G_{\alpha \beta}>G_{i j}\right) \backslash\{0\}, z \in E_{q}\left(G_{\alpha \beta}>G_{i j}\right)$. In the right hand side of the equation

$$
0=d(u)=-G_{i j} d y+p_{i j}^{*}(\Delta) y+d z
$$

the last two terms, $p_{i j}^{*}(\Delta) y$ and $d z$, the monomials in the canonical basis contain only factors $G_{\alpha \beta}>G_{i j}$; therefore $d y=0$ by induction on $q$, and $y=0$, and this gives a contradiction.

Proof of Proposition 1.2 The monomials from the canonical basis lying on the top horizontal line are $p_{1}^{*}\left(x_{h}\right) G_{12} G_{i_{2} 3} \cdots G_{i_{n-1} n}$, where $\left|x_{h}\right|=i \in[0,2 m]$ and $i_{a} \leq a$. 
The next composition is an isomorphism,

$$
\begin{aligned}
& E_{n-1}^{(n-1)(2 m-1)+i} \stackrel{d}{\longrightarrow} E_{n-2}^{(n-1)(2 m-1)+i+1} { \stackrel{p r}{\longrightarrow} \bigoplus_{ \stackrel { p r } { \longrightarrow } \bigoplus _ {\substack{ \substack {I_{*}={ I _ { * } = (\begin{subarray}{c}{i_{2}, \ldots, i_{n-1} \\
i_{a} \leq a} }} \\
{\longrightarrow}\end{subarray}} p_{1}^{*}\left(H^{i}\right) p_{2}^{*}\left(H^{2 m}\right) G_{i_{2} 3} \cdots G_{i_{n-1} n},} \\
& p_{1}^{*}\left(x_{h}\right) G_{12} G_{i_{2} 3} \cdots G_{i_{n-1} n} \mapsto p_{1}^{*}\left(x_{h}\right) p_{2}^{*}(w) G_{i_{2} 3} \cdots G_{i_{n-1} n},
\end{aligned}
$$

therefore the differential is injective on the top horizontal line.

\section{An acyclic subalgebra of the Križ model}

In [6] is introduced a quotient of the Križ model, denoted by $J_{n}$, which is quasiisomorphic to $E_{*}^{*}(X, n)$, but the corresponding kernel is not $\mathcal{S}_{n}$-stable. We will identify an acyclic subcomplex of $E_{*}^{*}(X, n)$, denoted by $E_{*}^{*}(w(X, n))$ (or simply by $E_{*}^{*}(w)$ ), which is also an $\mathcal{S}_{n}$-submodule and a subalgebra, giving another smaller complex quasi-isomorphic to the Križ model:

$$
S E_{*}^{*}(X, n)=E_{*}^{*} / E_{*}^{*}(w), \quad H^{*}\left(E_{*}^{*}(X, n)\right) \cong H^{*}\left(S E_{*}^{*}(X, n)\right)
$$

The last isomorphism is now $\mathcal{S}_{n}$-equivariant.

We start with a well known result in the theory of hyperplane arrangements, see for example Orlik and Terao [20]; a simple proof is included.

Definition 5.1 We define the differential $\partial$ of degree -1 on the Arnold algebra $\mathcal{A}^{*}(n)$ by $\partial G_{i j}=1$ and we call $\left(\mathcal{A}^{*}(n), \partial\right)$ the Arnold differential algebra.

Proposition 5.1 The Arnold differential algebra $\left(\mathcal{A}^{*}(n), \partial\right)$ is acyclic.

Proof Define the homotopy $h: \mathcal{A}^{*} \rightarrow \mathcal{A}^{*+1}$ by $h(\gamma)=G_{12} \gamma$ and verify that $\partial h+h \partial=\mathrm{id}_{\mathcal{A}^{*}}$.

We denote by $E_{*}^{\text {Top }}(X, n)$ the submodule of the Križ model $\left(E_{*}^{*}(X, n), d\right)$ given by the sum of the submodules of maximal total degree in each $q$-exterior degree

$$
E_{*}^{\mathrm{Top}}(X, n)=\bigoplus_{q=0}^{n-1} E_{q}^{\mathrm{Top}}(X, n)=\bigoplus_{q=0}^{n-1} E_{q}^{2 m n-q}(X, n) .
$$

It is obvious that $E_{*}^{\text {Top }}(X, n)$, the right side of the trapezoid, is a subcomplex and an ideal of $E_{*}^{*}(X, n)$. 
Proposition 5.2 There is an isomorphism of chain complexes of $\mathcal{S}_{n}$-modules

$$
\left(\mathcal{A}^{*}(n), \partial\right) \cong\left(E_{*}^{\mathrm{Top}}(X, n), d\right)
$$

In particular

$$
H^{*}\left(E_{*}^{\mathrm{Top}}(X, n), d\right)=0 .
$$

Proof Using the standard basis $\left\{G_{I_{*} J_{*}}=G_{i_{1} j_{1}} G_{i_{2} j_{2}} \cdots G_{i_{q} j_{q}}\right\}$ in $\mathcal{A}^{q}(n)$ and the basis $\left\{\prod_{h \notin J_{*}} p_{h}^{*}(w) G_{I_{*} J_{*}}\right\}$ in $E_{q}^{\mathrm{Top}}(X, n)$ (here $\left.2 \leq j_{1}<j_{2}<\cdots<j_{q} \leq n, 1 \leq i_{a}<j_{a}\right)$ we define the isomorphism

$$
f: \mathcal{A}^{q} \rightarrow E_{q}^{\mathrm{Top}}, \quad f\left(G_{I_{*} J_{*}}\right)=\prod_{h \notin J_{*}} p_{h}^{*}(w) G_{I_{*} J_{*}} .
$$

Obviously $f$ is $\mathcal{S}_{n}$-equivariant (the degree of $w$ is even) and $f$ preserves the differentials:

$$
\begin{aligned}
d f\left(G_{I_{*} J_{*}}\right) & =d\left(\prod_{h \notin J_{*}} p_{h}^{*}(w) G_{I_{*} J_{*}}\right) \\
& =\sum_{a=1}^{q}(-1)^{a+1} \prod_{h \notin J_{*}} p_{h}^{*}(w) \cdot p_{i_{a} j_{a}}^{*}(\Delta) G_{i_{1} j_{1}} \cdots \widehat{G_{i_{a} j_{a}}} \cdots G_{i_{q} j_{q}} \\
& =\sum_{a=1}^{q}(-1)^{a+1} \prod_{h \notin J_{*}} p_{h}^{*}(w) \cdot p_{j_{a}}^{*}(w) G_{i_{1} j_{1}} \cdots \widehat{G_{i_{a} j_{a}}} \cdots G_{i_{q} j_{q}} \\
& =\sum_{a=1}^{q}(-1)^{a+1} \prod_{h \notin J_{*} \backslash\left\{j_{a}\right\}} p_{h}^{*}(w) G_{i_{1} j_{1}} \cdots \widehat{G_{i_{a} j_{a}}} \cdots G_{i_{q} j_{q}} \\
& =f\left(\sum_{a=1}^{q}(-1)^{a+1} G_{i_{1} j_{1}} \cdots \widehat{G_{i_{a} j_{a}}} \cdots G_{i_{q} j_{q}}\right) \\
& =f \partial\left(G_{I_{*} J_{*}}\right)
\end{aligned}
$$

For the third equality we used $i_{a} \notin J_{*}$ and the equality

$$
p_{i_{a}}^{*}(w) p_{i_{a} j_{a}}^{*}(\Delta)=p_{i_{a}}^{*}(w) p_{j_{a}}^{*}(w) .
$$

Proof of Proposition 1.3 Now this is obvious.

Now we will define three acyclic subcomplexes which generalize the previous subcomplex $\left(E_{*}^{\text {Top }}(X, n), d\right)$. For a fixed nonempty subset $A \subset\{1,2, \ldots, n\}$ of cardinality $|A|=a \geq 2$ and a fixed sequence $\beta$ of length $b=n-a, \beta=\left(x_{1}, x_{2}, \ldots, x_{b}\right)$, where 
all the elements $x_{j}$ belong to the fixed basis $\mathcal{B}$ and are different from $w$, we denote the increasing sequence of elements in $\{1,2, \ldots, n\} \backslash A$ by $b_{1}<b_{2}<\cdots<b_{b}$, the product $\prod_{j=1}^{b} p_{b_{j}}^{*}\left(x_{j}\right)$ by $p^{*}(\beta)$, and its degree $\sum_{j=1}^{b} \operatorname{deg}\left(x_{j}\right)$ by $|\beta|$. Now we define the subspace

$$
E_{*}^{\mathrm{Top}}(A, \beta)=\sum_{q=0}^{a-1} E_{q}^{2 m a-q+|\beta|}(A, \beta)
$$

by

$$
E_{q}^{2 m a-q+|\beta|}(A, \beta)=\mathbb{Q}\left\langle\prod_{i \in A \backslash J_{*}} p_{i}^{*}(w) p^{*}(\beta) G_{I_{*} J_{*}}\left|I_{*} \cup J_{*} \subset A,\right| J_{*} \mid=q\right\rangle
$$

(in words: the scalars in the "complementary positions," given by $\beta$, should be different from $w$, on the "forbidden positions," corresponding to $J_{*}$, there is only 1 , and all the other "possible positions" should be filled with the top class $w$ ).

Proposition 5.3 For any $A$ and $\beta$ as before, the space $E_{*}^{\text {Top }}(A, \beta)$ is an acyclic subcomplex of the Križ model.

Proof By definition $E_{*}^{\text {Top }}(A, \beta)$ is the direct sum of its subspaces $E_{q}^{2 m a-q+|\beta|}(A, \beta)$ and it is stable under the differential:

$$
\begin{aligned}
d\left(\prod_{i \in A \backslash J_{*}} p_{i}^{*}(w) p^{*}(\beta) G_{I_{*} J_{*}}\right) & =\sum_{j_{h} \in J_{*}} \pm \prod_{i \in A \backslash J_{*}} p_{i}^{*}(w) p_{j_{h}}^{*}(w) p^{*}(\beta) G_{I_{*} \backslash\left\{i_{h}\right\} J_{*} \backslash\left\{j_{h}\right\}} \\
& =\sum_{j_{h} \in J_{*}} \pm \prod_{i \in A \backslash\left(J_{*} \backslash\left\{j_{h}\right\}\right)} p_{i}^{*}(w) p^{*}(\beta) G_{I_{*} \backslash\left\{i_{h}\right\} J_{*} \backslash\left\{j_{h}\right\}}
\end{aligned}
$$

This subcomplex is acyclic because of the isomorphism

$$
\left(E_{*}^{\mathrm{Top}}(A, \beta), d\right) \cong\left(\mathcal{A}^{*}(a), \partial\right)
$$

given by

$$
\prod_{i \in A \backslash J_{*}} p_{i}^{*}(w) p^{*}(\beta) G_{I_{*} J_{*}} \longleftrightarrow(-1)^{\beta} G_{I_{*} J_{*}},
$$

the differential of $\beta$ is zero and the compatibility of the differentials $d, \partial$ was checked in the proof of 5.2.

Example 5.2 If $A=\{1,2, \ldots, n\}$, then $\beta$ is the empty sequence, $p^{*}(\beta)=1$ and $E_{*}^{\text {Top }}(\{1,2, \ldots, n\}, \phi)=E_{*}^{\text {Top }}(X, n)$. 
Now we fix a number $a$ from 2 to $n$ and a sequence $\beta=\left(x_{1}, \ldots, x_{b}\right)$ of length $b=n-a$ as before. We say that the sequence $\beta^{\prime}=\left(y_{1}, \ldots, y_{b}\right)$ is similar to $\beta$, $\beta \sim \beta^{\prime}$, if there is a permutation $\sigma \in \mathcal{S}_{b}$ such that $y_{i}=x_{\sigma(i)}$ for $i=1,2, \ldots, b$. We define a new subspace

$$
E_{*}^{\mathrm{Top}}(a, \beta)=\sum_{|A|=a, \beta^{\prime} \sim \beta} E_{*}^{\mathrm{Top}}\left(A, \beta^{\prime}\right) .
$$

Proposition 5.4 For any number $a$ and a sequence $\beta$ as before, the space $E_{*}^{\text {Top }}(a, \beta)$ is an acyclic subcomplex and $\mathcal{S}_{n}$-invariant.

Proof The space is $\mathcal{S}_{n}$-invariant by construction,

$$
\sigma\left(E_{*}^{\mathrm{Top}}(A, \beta)\right)=E_{*}^{\mathrm{Top}}(\sigma A, \sigma \beta)=E_{*}^{\mathrm{Top}}\left(\sigma A, \beta^{\prime}\right),
$$

and the acyclicity is a consequence of the direct sum decomposition

$$
\left(E_{*}^{\mathrm{Top}}(a, \beta), d\right)=\bigoplus_{|A|=a, \beta^{\prime} \sim \beta}\left(E_{*}^{\mathrm{Top}}\left(A, \beta^{\prime}\right), d\right) .
$$

Finally, we take the whole collection of these subcomplexes,

$$
E_{*}^{*}(w(X, n))=\sum_{a=2}^{n} \sum_{\substack{\text { of length } \\ n-a}} E_{*}^{\mathrm{Top}}(a, \beta) .
$$

Proposition 5.5 The space $E_{*}^{*}(w(X, n))$ is an acyclic, $\mathcal{S}_{n}$-invariant subcomplex.

Proof It suffices to show the double sum is a direct sum: a monomial $x_{1} \otimes \cdots \otimes x_{n} G_{I_{*} J_{*}}$ from the canonical basis in $E_{*}^{*}(w(X, n))$ defines in a unique way the subset $A$ and the factor $\beta$ :

$$
\begin{aligned}
A & =\left\{i \in\{1,2, \ldots, n\} \mid x_{i}=w\right\} \cup J_{*}, \\
\beta & =\left(x_{h_{1}}, x_{h_{2}}, \ldots, x_{h_{b}}\right),
\end{aligned}
$$

where $h_{1}<h_{2}<\cdots<h_{b}$ are the elements of $\{1,2, \ldots, n\} \backslash A$.

Proposition 5.6 The projection map

$$
E_{*}^{*}(X, n) \longrightarrow S E_{*}^{*}(X, n)=E_{*}^{*}(X, n) / E_{*}^{*}(w(X, n))
$$

is a quasi-isomorphism.

Proof This is obvious from the long exact sequence associated to

$$
0 \longrightarrow E_{*}^{*}(w(X, n)) \longrightarrow E_{*}^{*}(X, n) \longrightarrow S E_{*}^{*}(X, n) \longrightarrow 0 .
$$




\section{An example: $F\left(\mathbb{C P} P^{1}, n\right)$}

We analyze the cohomology algebra of the configuration space of the complex projective line using the symmetric structure of the Križ model. We encode the symmetric structure of a bigraded $\mathcal{S}_{n}$-module $H_{*}^{*}$ into the $\mathcal{S}_{n}$-Poincaré polynomial:

$$
S P_{H_{*}^{*}}(t, s)=\sum_{\lambda \vdash n}\left(\sum_{k, q} m_{q, \lambda}^{k} t^{k} s^{q}\right) V(\lambda),
$$

where we have that $m_{q, \lambda}^{k}$ is the multiplicity of the irreducible representation $V(\lambda)$ in the component $H_{q}^{k}$; the double Poincaré polynomial of $H_{*}^{*}$ is a consequence of $S P_{H_{*}^{*}}$ : $P_{H_{*}^{*}}(t, s)=\sum_{k, q}\left(\sum_{\lambda \vdash n} m_{q, \lambda}^{k} \operatorname{dim} V(\lambda)\right) t^{k}{ }^{q} q$.

For $n=2$ and $n=3$ we have the next tables of the symmetric group structure of the Križ model; using the injectivity properties of the differential, we obtain the first table and for the second table we have to use the vanishing of the cohomology on the left, top and the right side and also the acyclicity of the "interior part" $\bigoplus_{|A|=2} E_{*}^{\text {Top }}(A, 1)$ :

$$
\begin{aligned}
V(3) \oplus V(2,1) & \cong\left\langle w \otimes 1 \otimes 1 G_{12}, w \otimes 1 \otimes 1 G_{13}, 1 \otimes w \otimes 1 G_{23}\right\rangle \\
& \stackrel{d}{\cong}\langle w \otimes w \otimes 1, w \otimes 1 \otimes w, 1 \otimes w \otimes w\rangle
\end{aligned}
$$
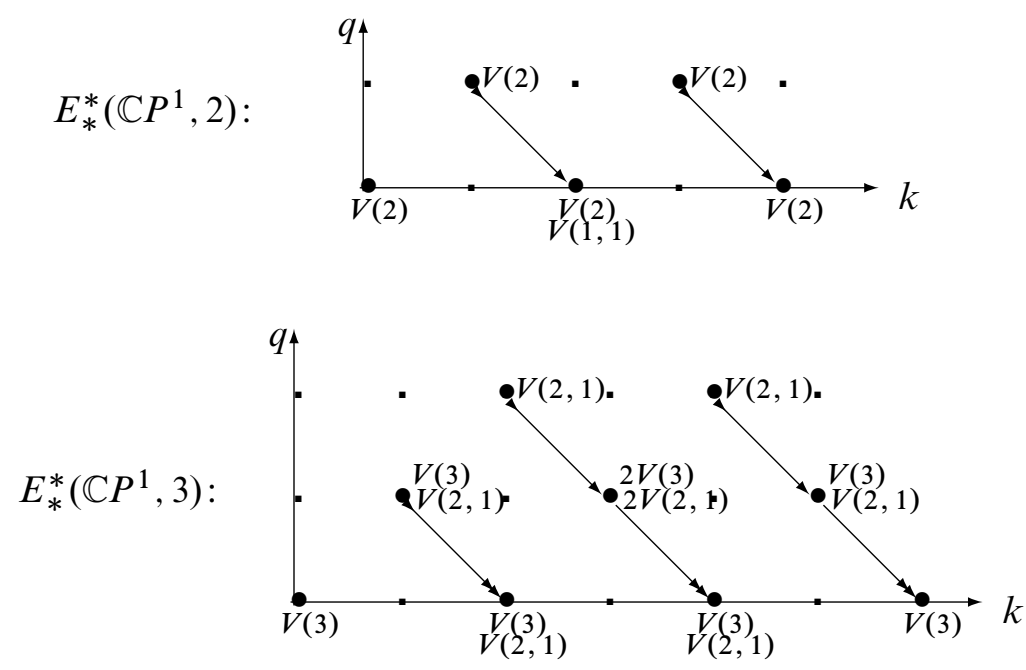

As a consequence we obtain the following. 
Lemma 6.1 The nonzero components of the cohomology algebra of $F\left(\mathbb{C} P^{1}, 2\right)$ and $F\left(\mathbb{C} P^{1}, 3\right)$ are

$$
\begin{aligned}
& H_{0}^{0}\left(F\left(\mathbb{C} P^{1}, 2\right)\right) \cong V(2), \quad H_{0}^{2}\left(F\left(\mathbb{C} P^{1}, 2\right)\right) \cong V(1,1), \\
& H_{0}^{0}\left(F\left(\mathbb{C} P^{1}, 3\right)\right) \cong V(3), \quad H_{1}^{3}\left(F\left(\mathbb{C} P^{1}, 3\right)\right) \cong V(3) .
\end{aligned}
$$

In particular their symmetric Poincaré polynomials are

$$
\begin{aligned}
& P_{F\left(\mathbb{C} P^{1}, 2\right)}(t, s)=V(2)+t^{2} V(1,1), \\
& P_{F\left(\mathbb{C} P^{1}, 3\right)}(t, s)=\left(1+s t^{3}\right) V(3) .
\end{aligned}
$$

Corollary 6.2 The Poincare polynomials of the unordered configuration spaces of the projective line are

$$
\begin{aligned}
& P_{C\left(\mathbb{C} P^{1}, 2\right)}(t)=1, \\
& P_{C\left(\mathbb{C} P^{1}, 3\right)}(t)=1+t^{3} .
\end{aligned}
$$

Another consequence of the last computation is the fact that the Serre spectral sequences of the fibrations

$$
\mathcal{F}_{n}: F(\mathbb{C}, n-1) \hookrightarrow F\left(\mathbb{C} P^{1}, n\right) \rightarrow \mathbb{C} P^{1}
$$

do not degenerate at $\boldsymbol{E}_{2}^{*, *}$ (for $n \geq 3$ ): using the vanishing of the first and second cohomology of $F\left(\mathbb{C} P^{1}, 3\right)$ and the projection $p: F\left(\mathbb{C} P^{1}, n\right) \rightarrow F\left(\mathbb{C} P^{1}, 3\right)$, we obtain the diagram (we use bold $\boldsymbol{E}_{*}^{*, *}$ for the components in the spectral sequences):

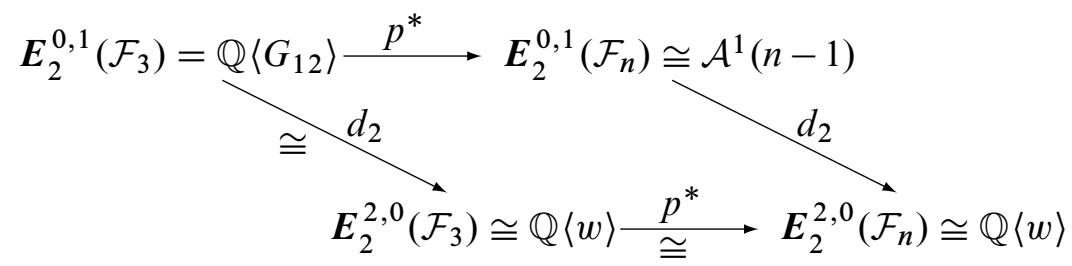

and we find that the differential $d_{2}$ is surjective for $n \geq 3$. These spectral sequences degenerate at $\boldsymbol{E}_{3}$ : the two nonzero columns are given by

$$
\begin{gathered}
\boldsymbol{E}_{\infty}^{0, *}=\boldsymbol{E}_{3}^{0, *}=\operatorname{ker} d_{2} \cong \mathcal{A}^{*}(n-1) /\left(G_{12}\right), \\
\boldsymbol{E}_{\infty}^{2, *}=\boldsymbol{E}_{3}^{2, *} \cong \boldsymbol{E}_{2}^{2, *} / \operatorname{Im} d_{2} \cong \mathbb{Q}\left\langle w G_{12}\right\rangle \otimes \mathcal{A}^{*}(n-1) /\left(G_{12}\right) .
\end{gathered}
$$

Proposition 6.1 [13] The cohomology algebra of the configuration space $F\left(\mathbb{C} P^{1}, n\right)$ $(n \geq 3)$ is given by

$$
H^{*}\left(F\left(\mathbb{C} P^{1}, n\right)\right) \cong H^{*}\left(F\left(\mathbb{C} P^{1}, 3\right)\right) \otimes \mathcal{A}^{*}(n-1) /\left(G_{12}\right) .
$$


In particular, its Poincaré polynomial is

$$
P_{F\left(\mathbb{C} P^{1}, n\right)}(t)=\left(1+t^{3}\right)(1+2 t)(1+3 t) \cdots(1+(n-2) t) .
$$

Using the results from Section 5, we detect the nonzero bigraded components of the cohomology algebra and (partially) its $\mathcal{S}_{n}$-structure.

Proof of Theorem 1.2 The first cohomology group is

$$
H^{1}\left(F\left(\mathbb{C} P^{1}, n\right)\right)=H_{1}^{1} \cong V(n-2,2),
$$

and the subalgebra generated by degree 1 elements is contained in $\bigoplus_{q=0}^{n-3} H_{q}^{q}$. The element

$$
\gamma=2(n-2) \sum_{i<j} p_{i}^{*}(w) G_{i j}-\sum_{i<j} \sum_{k \neq i, j} p_{k}^{*}(w) G_{i j} \in E_{1}^{3}\left(\mathbb{C} P^{1}, n\right)
$$

is a cocycle in the $V(n)$-isotypic component. It can not be a coboundary because $V(n)$ is missing from $E_{2}^{2}$ :

$$
\begin{aligned}
E_{2}^{2}\left(\mathbb{C} P^{1}, n\right) & \cong \mathcal{A}^{2}(n) \\
& \cong 2 V(1)_{n} \oplus 2 V(2)_{n} \oplus 2 V(1,1)_{n} \oplus V(3)_{n} \oplus 2 V(2,1)_{n} \oplus V(3,1)_{n}
\end{aligned}
$$

(this is correct in the stable case $n \geq 7$ (see [9] or [2]); the trivial module $V(n)$ does not appear in the unstable cases either).

As $\beta_{3}=1+\sum_{2 \leq i<j<k \leq n-2} i j k$ and the component $H_{3}^{3}$ contains a submodule of dimension $\beta_{3}-1$, we obtain

$$
H_{1}^{3}\left(F\left(\mathbb{C} P^{1}, n\right)\right) \cong V(n) .
$$

The ideal generated by $\gamma$ is contained in $\bigoplus_{q=1}^{n-2} H_{q}^{q+2}$ and algebra structure shows that all the other bigraded components are zero.

The module $H_{2}^{2}$ is a quotient of $E_{2}^{2} \cong \mathcal{A}^{2}(n)$ (its decomposition into irreducible modules was given in the last proof) and also a quotient of

$$
\begin{aligned}
H_{1}^{1} \wedge H_{1}^{1} & \cong \bigwedge^{2} V(2)_{n} \\
& \cong V(1,1)_{n} \oplus V(2,1)_{n} \oplus V(1,1,1)_{n} \oplus V(3,1)_{n}
\end{aligned}
$$

(see [2]); the intersection of these decompositions gives (for $n \geq 7$ ) the inclusion

$$
H_{2}^{2}<V(1,1)_{n} \oplus V(2,1)_{n} \oplus V(3,1)_{n}
$$


and computing their dimensions this inclusion becomes an equality:

$$
\begin{aligned}
\beta_{2} & =\sum_{2 \leq i<j \leq n-2} i j=\frac{(n-4)(n-3)\left(3 n^{2}-n+2\right)}{24} \\
& =\frac{(n-1)(n-2)}{2}+\frac{n(n-1)(n-4)}{3}+\frac{n(n-1)(n-3)(n-6)}{8} \\
& =\operatorname{dim} V(1,1)_{n}+\operatorname{dim} V(2,1)_{n}+\operatorname{dim} V(3,1)_{n}
\end{aligned}
$$

Similar computations give the unstable cases of the next proposition.

Proposition 6.2 The decomposition of the second cohomology group becomes stable for $n \geq 7$ and it is given by

$$
H^{2}\left(F\left(\mathbb{C} P^{1}, n\right)\right)=H_{2}^{2} \cong V(1,1)_{n} \oplus V(2,1)_{n} \oplus V(3,1)_{n} .
$$

In the unstable cases we have

$$
\begin{aligned}
& H^{2}\left(F\left(\mathbb{C} P^{1}, n\right)\right)=0, \text { for } n=2,3,4, \\
& H^{2}\left(F\left(\mathbb{C} P^{1}, 5\right)\right) \cong V(3,1,1), \\
& H^{2}\left(F\left(\mathbb{C} P^{1}, 6\right)\right) \cong V(4,1,1) \oplus V(3,2,1) .
\end{aligned}
$$

\section{References}

[1] V I Arnold, The cohomology ring of the group of dyed braids, Mat. Zametki 5 (1969) 227-231 MR0242196

[2] S Ashraf, H Azam, B Berceanu, Representation stability of power sets and square free polynomials arXiv:1106.4926v1

[3] S Ashraf, B Berceanu, Cohomology of 3-points configuration spaces of complex projective spaces arXiv:1212.1291v1

[4] S Ashraf, B Berceanu, Equivariant Lefschetz structure of the Križ model, in preparation

[5] H Azam, B Berceanu, Cohomology of configuration spaces of Riemann surfaces, in preparation

[6] B Berceanu, M Markı, S Papadima, Multiplicative models for configuration spaces of algebraic varieties, Topology 44 (2005) 415-440 MR2114955

[7] G M Bergman, The diamond lemma for ring theory, Adv. in Math. 29 (1978) 178-218 MR506890

[8] R Bezrukavnikov, Koszul DG-algebras arising from configuration spaces, Geom. Funct. Anal. 4 (1994) 119-135 MR1262702 
[9] T Church, B Farb, Representation theory and homological stability, Adv. Math. 245 (2013) 250-314 MR3084430

[10] F R Cohen, L R Taylor, Computations of Gel' fand-Fuks cohomology, the cohomology of function spaces, and the cohomology of configuration spaces, from: "Geometric applications of homotopy theory, I”, (M G Barratt, ME Mahowald, editors), Lecture Notes in Math. 657, Springer, Berlin (1978) 106-143 MR513543

[11] F R Cohen, L R Taylor, Configuration spaces: Applications to Gelfand-Fuks cohomology, Bull. Amer. Math. Soc. 84 (1978) 134-136 MR0461522

[12] P Deligne, P Griffiths, J Morgan, D Sullivan, Real homotopy theory of Kähler manifolds, Invent. Math. 29 (1975) 245-274 MR0382702

[13] E M Feichtner, G M Ziegler, The integral cohomology algebras of ordered configuration spaces of spheres, Doc. Math. 5 (2000) 115-139 MR1752611

[14] W Fulton, J Harris, Representation theory, Graduate Texts in Mathematics 129, Springer, New York (1991) MR1153249

[15] W Fulton, R MacPherson, A compactification of configuration spaces, Ann. of Math. 139 (1994) 183-225 MR1259368

[16] I Križ, On the rational homotopy type of configuration spaces, Ann. of Math. 139 (1994) 227-237 MR1274092

[17] P Lambrechts, D Stanley, A remarkable DG module model for configuration spaces, Algebr. Geom. Topol. 8 (2008) 1191-1222 MR2443112

[18] W Ledermann, Introduction to group characters, 2nd edition, Cambridge Univ. Press (1987) MR901944

[19] G I Lehrer, L Solomon, On the action of the symmetric group on the cohomology of the complement of its reflecting hyperplanes, J. Algebra 104 (1986) 410-424 MR866785

[20] P Orlik, H Terao, Arrangements of hyperplanes, Grundl. Math. Wissen. 300, Springer, Berlin (1992) MR1217488

[21] J-P Serre, Linear representations of finite groups, Graduate Texts in Mathematics 42, Springer, New York (1977) MR0450380

[22] R P Stanley, Some aspects of groups acting on finite posets, J. Combin. Theory Ser. A 32 (1982) 132-161 MR654618

SA, HA, BB: Abdus Salam School of Mathematical Sciences, GC University 68-B New Muslim Town, Lahore-54600, Pakistan

BB: Institute of Mathematics Simion Stoilow

PO Box 1-764, RO-014700 Bucharest, Romania

samia.ashraf@yahoo.com, centipedes.united@gmail.com,

Barbu.Berceanu@imar.ro

Received: 4 May 2012 Revised: 4 July 2013 